\title{
A numerical method for Mean Field Games on networks
}

\author{
Simone Cacace, Fabio Camilli ${ }^{\dagger}$ and Claudio Marchi ${ }^{\ddagger}$
}

\begin{abstract}
We propose a numerical method for stationary Mean Field Games defined on a network. In this framework a correct approximation of the transition conditions at the vertices plays a crucial role. We prove existence, uniqueness and convergence of the scheme and we also propose a least squares method for the solution of the discrete system. Numerical experiments are carried out.
\end{abstract}

AMS subject classification: 91A15, 35R02, 35B30, 49N70, 65M06.

Keywords: networks, mean field games, finite difference schemes, convergence.

\section{Introduction}

The Mean Field Game (MFG in short) theory has been introduced in [16, 18] to describe the limit behavior of differential games when the number of agents becomes very large. Models based on this theory can be used to investigate crowd dynamics, consensus formation and various economical and social problems (as growth theory, environmental policy and formation of volatility in financial markets) in which the strategy of the single agent determines a collective behavior of the population (see [4, 12, 15]).

From a mathematical point of view, MFG theory leads to the study of a coupled system of two differential equations: a Hamilton-Jacobi-Bellman equation and a FokkerPlanck equation, describing respectively the optimal behavior of each single agent and the evolution of the whole population. There is a rapidly increasing literature concerning both the theoretical aspects and the applications of MFG (see the review paper [13]).

A crucial point to extend the theory of MFG systems to networks is to find the appropriate transition conditions at the vertices in order to obtain a well posed mathematical problem, coherent with the applications. In [8], it was considered a MFG system with quadratic Hamiltonian which, by an appropriate change of variable, can be transformed into a linear system of differential equations coupled only via the initial datum. A general class of stationary MFG systems on networks is considered in [7, where it is proved existence and uniqueness of classical solutions to the problem

$$
\begin{cases}-\nu \partial^{2} u+H(x, \partial u)+\lambda=V[m], & x \in \Gamma \\ \nu \partial^{2} m+\partial\left(m H_{p}(x, \partial u)\right)=0, & x \in \Gamma \\ \int_{\Gamma} m(x) d x=1, \int_{\Gamma} u(x) d x=0 . & \end{cases}
$$

\footnotetext{
${ }^{*}$ Dip. di Matematica, "Sapienza" Università di Roma, p.le A. Moro 5, 00185 Roma, Italy, (e-mail: cacace@mat.uniroma1.it)

†Dip. di Scienze di Base e Applicate per l'Ingegneria, "Sapienza" Università di Roma, via Scarpa 16, 00161 Roma, Italy, (e-mail: camilli@sbai.uniroma1.it)

${ }^{\ddagger}$ Dip. di Ingegneria dell’Informazione, Università di Padova, via Gradenigo 6/B, 35131 Padova, Italy (e-mail: claudio.marchi@unipd.it)
} 
Here the network $\Gamma=(\mathcal{V}, \mathcal{E})$ is a finite collection of points $\mathcal{V}:=\left\{v_{i}\right\}_{i \in I}$ in $\mathbb{R}^{n}$ indexed by $I$, connected by continuous, non self-intersecting $\operatorname{arcs} \mathcal{E}:=\left\{e_{j}\right\}_{j \in J}$ indexed by $J$. Moreover, $\nu=\left(\nu_{j}\right)_{j \in J}$ are strictly positive numbers, the Hamiltonian $H$ is a collection $\left\{H_{j}\right\}_{j \in J}$ where $H_{j}$ are continuous, convex Hamiltonians defined on the arcs $e_{j}$, and $H_{p}(x, q)$ is the differential of $p \mapsto H(x, p)$ at $p=q$. Let us stress that $H$ may be discontinuous at the vertices.

The equations in (1.1), which have the same interpretation as in the classical MFG theory, are defined in terms of the coordinate parametrizing the arc $e_{j}, j \in J$, and have to be complemented with appropriate conditions at the vertices. At each internal vertex $v_{i}$ we consider the transition conditions

$$
\begin{aligned}
& \sum_{j \in \operatorname{Inc}_{i}} \nu_{j} \partial_{j} u\left(v_{i}\right)=0, \\
& \sum_{j \in \operatorname{Inc}_{i}}\left[\nu_{j} \partial_{j} m\left(v_{i}\right)+H_{j, p}\left(v_{i}, \partial_{j} u\right) m_{j}\left(v_{i}\right)\right]=0, \\
& u_{j}\left(v_{i}\right)=u_{k}\left(v_{i}\right), m_{j}\left(v_{i}\right)=m_{k}\left(v_{i}\right), \quad j, k \in \operatorname{Inc}_{i}
\end{aligned}
$$

where $\mathrm{Inc}_{i}$ denotes the set of the edges incident the vertex $v_{i}$ and $H_{j, p}$ is the derivative with respect to $p$ of the Hamiltonian defined on the edge $e_{j}$. We mention, see [7] for more details, that the first condition in 1.2 is the classical Kirchhoff condition and it prescribes the probability that an agent reaching the vertex $v_{i}$ enters in the incident edge $e_{j}, j \in \mathrm{Inc}_{i}$; the second condition in (1.2) guarantees the mass conservation at $v_{i}$ (the sum of the fluxes at $v_{i}$ is null); the third condition is the continuity of $u$ and $m$ at $v_{i}$. We remark that (1.2) are natural conditions for $2^{\text {nd }}$ order problems on networks. In fact the domain of the Laplace operator on a network is given by continuous functions on $\Gamma$ which are $H^{2}$ on the edges and which satisfy the Kirchhoff condition at the vertices [21]. Moreover, the transition conditions are a crucial ingredient for the validity of the maximum principle on networks.

In this paper we consider the numerical approximation of the problem (1.1)-(1.2) following the approach in [1, 2, where a finite difference approximation of the MFG system is studied (see also [9, 14, 17] for different approaches). Inside the edges we follow the same approach of [2] and we discretize the differential equations in (1.1) by finite differences. The guideline to find the correct approximation of the transition conditions in $(1.2)$ is to reproduce at a discrete level some fundamental identities which are obtained in the continuous setting by the weak formulation of the problem (see f.e. $(3.22)$ ). For this reason the discrete Hamiltonian defined by a monotone approximation of the HamiltonJacobi-Bellman equation is also used in the discretization of the Fokker-Planck equation and of the corresponding transition condition. By means of the previous identities we prove the well-posedness of the discrete problem and the convergence to the solution of the system 1.1.

While there is a large literature about the approximation of hyperbolic problems on networks (see for example [5], [10]), as far as we know, numerical schemes for second order differential equations on networks with Kirchhoff conditions have been only considered in the linear case (see [19], [20]). Hence the part concerning the approximation of the Hamilton-Jacobi-Bellman equation on the network is new and of independent interest.

The paper is organized as follows. In Section 2 we introduce assumptions and notations. Section 3 includes three subsections concerning existence, uniqueness and con- 
vergence. In Section 4 we present a method for the solution of the discrete system and some numerical examples illustrating the theory.

\section{Notations and preliminary definitions}

A network is a couple $(\mathcal{V}, \mathcal{E})$ given by a finite collection of vertices $\mathcal{V}:=\left\{v_{i}\right\}_{i \in I}$ and a finite collection $\mathcal{E}:=\left\{e_{j}\right\}_{j \in J}$ of continuous non self-intersecting arcs whose endpoints belong to $\mathcal{V}$. We assume that each $\operatorname{arc} e_{j} \in \mathcal{E}$ is parametrized by a smooth function $\pi_{j}:\left[0, l_{j}\right] \rightarrow \mathbb{R}^{n}, l_{j}>0$. For a function $u: \Gamma \rightarrow \mathbb{R}$ we denote by $u_{j}:\left[0, l_{j}\right] \rightarrow \mathbb{R}$ the restriction of $u$ to $e_{j}$, i.e. $u(x)=u_{j}(y)$ for $x \in e_{j}, y=\pi_{j}^{-1}(x)$. Given $v_{i} \in \mathcal{V}$, we denote by $\partial_{j} u\left(v_{i}\right)$ the oriented derivative at $v_{i}$ along the arc $e_{j}$ defined by

$$
\partial_{j} u\left(v_{i}\right)= \begin{cases}\lim _{t \rightarrow 0^{+}}\left(u_{j}(t)-u_{j}(0)\right) / t, & \text { if } v_{i}=\pi_{j}(0) ; \\ \lim _{t \rightarrow 0^{+}}\left(u_{j}\left(l_{j}-t\right)-u_{j}\left(l_{j}\right)\right) / t, & \text { if } v_{i}=\pi_{j}\left(l_{j}\right) .\end{cases}
$$

Given a discretization step $h=\left\{h_{j}\right\}_{j \in J}$, we consider an uniform partition $y_{j, k}=k h_{j}$, $k=0, \ldots, N_{j}^{h}$, of the interval $\left[0, l_{j}\right]$ which parameterizes the edge $e_{j}$ (we assume that $N_{j}^{h}=l_{j} / h_{j}$ is an integer). We obtain a spatial grid on $\Gamma$ by setting

$$
\mathcal{G}_{h}=\left\{x_{j, k}=\pi_{j}\left(y_{j, k}\right), j \in J, k=0, \ldots, N_{j}^{h}\right\} .
$$

We define

$$
\operatorname{Inc}_{i}^{+}=\left\{j \in \operatorname{Inc}_{i}: v_{i}=\pi_{j}(0)\right\}, \quad \operatorname{Inc}_{i}^{-}=\left\{j \in \operatorname{Inc}_{i}: v_{i}=\pi_{j}\left(N_{j}^{h} h_{j}\right)\right\},
$$

so that

$$
\operatorname{Inc}_{i}=\operatorname{Inc}_{i}^{+} \cup \operatorname{Inc}_{i}^{-},
$$

as shown in Figure 1 .

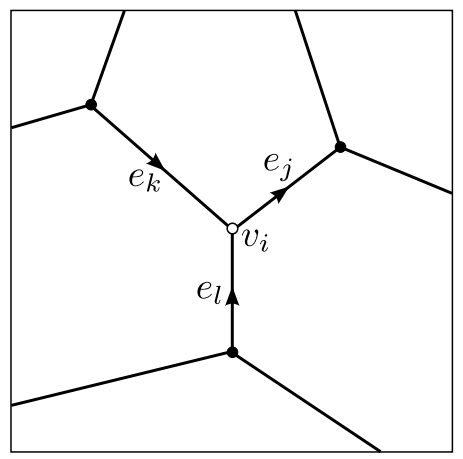

Figure 1: incident edges to the vertex $v_{i}: \operatorname{Inc}_{i}^{+}=\{j\}, \operatorname{Inc}_{i}^{-}=\{k, l\}$.

We set

$$
|h|=\max _{j \in J}\left\{h_{j}\right\}, \quad h_{v_{i}}=\sum_{j \in \operatorname{Inc}_{i}} \frac{h_{j}}{2}, \quad N^{h}=\#(I)+\sum_{j \in J}\left(N_{j}^{h}-1\right),
$$

i.e. $N^{h}$ is the total number of the points of $\mathcal{G}_{h}$, having identified for each $i \in I$ the $\#\left(\mathrm{Inc}_{i}\right)$ grid points corresponding to the same vertex $v_{i}$. For a grid function $U: \mathcal{G}_{h} \rightarrow \mathbb{R}$ we denote by $U_{j, k}$ its value at the grid point $x_{j, k}$. 
Definition 2.1 We say that a grid function $U: \mathcal{G}_{h} \rightarrow \mathbb{R}$ is continuous at $v_{i}$ if $U_{j, \ell}=U_{k, m}:=U_{i} \quad$ if $v_{i}=\pi_{j}\left(\ell h_{j}\right)=\pi_{k}\left(m h_{k}\right), j, k \in I n c_{i}, \ell \in\left\{0, N_{j}^{h}\right\}$, and $m \in\left\{0, N_{k}^{h}\right\}$, i.e. the value of $U$ at the vertex $v_{i}$ is independent of incident edge $e_{j}, j \in$ Inc $_{i}$. We say that a a grid function is continuous if it is continuous at $v_{i}$, for each $i \in I$.

We introduce the finite difference operators

$$
\begin{aligned}
& \left(D^{+} U\right)_{j, k}=\frac{U_{j, k+1}-U_{j, k}}{h_{j}}, \\
& {\left[D_{h} U\right]_{j, k}=\left(\left(D^{+} U\right)_{j, k},\left(D^{+} U\right)_{j, k-1}\right)^{T},} \\
& \left(D_{h}^{2} U\right)_{j, k}=\frac{U_{j, k-1}-2 U_{j, k}+U_{j, k+1}}{h_{j}^{2}} .
\end{aligned}
$$

In order to approximate the Hamiltonian $H_{j}:\left[0, l_{j}\right] \times \mathbb{R} \rightarrow \mathbb{R}, j \in J$, we consider a numerical Hamiltonian $g_{j}:\left[0, l_{j}\right] \times \mathbb{R}^{2} \rightarrow \mathbb{R},\left(x, q_{1}, q_{2}\right) \rightarrow g_{j}\left(x, q_{1}, q_{2}\right)$ satisfying the following assumptions:

(G1) monotonicity: $g_{j}$ is nonincreasing with respect to $q_{1}$ and nondecreasing with respect to $q_{2}$.

(G2) consistency: $g_{j}(x, q, q)=H_{j}(x, q) \forall x \in\left[0, l_{j}\right], \forall q \in \mathbb{R}$.

(G3) differentiability: $g_{j}$ is of class $\mathcal{C}^{1}$.

(G4) superlinear growth : $g_{j}\left(x, q_{1}, q_{2}\right) \geq \alpha\left(\left(q_{1}^{-}\right)^{2}+\left(q_{2}^{+}\right)^{2}\right)^{\gamma / 2}-C$ for some $\alpha>0, C \in \mathbb{R}$, $\gamma>1$ and $q_{s}^{ \pm}$denote the positive and negative part of $q_{s}, s=1,2$

(G5) convexity : for all $x \in e_{j},\left(q_{1}, q_{2}\right) \mapsto g_{j}\left(x, q_{1}, q_{2}\right)$ is convex.

Numerical Hamiltonians fulfilling these requirements are provided by Lax-Friedrichs or Godunov type schemes, see [22]. As an example, suppose that the Hamiltonian $H$ is of the form $H(x, p)=\Psi(x,|p|)$ where $\Psi$ is convex, increasing and superlinear with respect to its second argument. Then the Engquist-Osher Godunov scheme reads as

$$
g_{j}\left(x, q_{1}, q_{2}\right)=\Psi\left(x,\left(\min \left(q_{1}, 0\right)^{2}+\max \left(q_{2}, 0\right)\right)^{2}\right)
$$

and the monotonicity, consistency and coercivity conditions are satisfied.

Given $U, W: \mathcal{G}_{h} \rightarrow \mathbb{R}$, we define the scalar product

$$
(U, W)_{2}=\sum_{j \in J} \sum_{k=1}^{N_{j}^{h}-1} h_{j} U_{j, k} W_{j, k}+\sum_{i \in I}\left(\sum_{j \in \operatorname{Inc}_{i}^{+}} \frac{h_{j}}{2} U_{j, 0} W_{j, 0}+\sum_{j \in \operatorname{Inc}_{i}^{-}} \frac{h_{j}}{2} U_{j, N_{j}^{h}} W_{j, N_{j}^{h}}\right) .
$$

We introduce the compact and convex set

$$
\mathcal{K}_{h}=\left\{\left(M_{j, k}\right)_{j \in J, 0 \leq k \leq N_{j}^{h}}: M \text { is continuous, } M_{j, k} \geq 0,(M, 1)_{2}=1\right\} .
$$

The operator $V[m]\left(x_{j, k}\right)$ is approximated by $\left(V_{h}[M]\right)_{j, k}$ where $M$ is the piecewise constant function taking the value $M_{j, k}$ in the interval $\left|y-y_{j, k}\right| \leq h_{j} / 2, k=1, \ldots, N_{j}^{h}-1$, $j \in J$ (at the vertices only the half interval contained in $\left[0, l_{j}\right]$ is considered). In particular, if $V$ is a local operator, i.e. $V[m](x)=F(m(x))$, then we set $\left(V_{h}[M]\right)_{j, k}=F\left(M_{j, k}\right)$. We assume that 
(V1) $V_{h}$ is continuous and maps $\mathcal{K}_{h}$ on a bounded set of grid functions.

(V2) $V_{h}$ is monotone, i.e.

$$
\left(V_{h}[M]-V_{h}[\bar{M}], M-\bar{M}\right)_{2} \leq 0 \Rightarrow M=\bar{M} .
$$

(V3) There exists $C$ independent of $h$ such that for all grid functions $M \in \mathcal{K}_{h}$

$$
\begin{aligned}
& \left\|V_{h}[M]\right\|_{\infty}:=\max _{j, k}\left|\left(V_{h}[M]\right)_{j, k}\right| \leq C \\
& \left|\left(V_{h}[M]\right)_{j, k}-\left(V_{h}[M]\right)_{j, \ell}\right| \leq C\left|y_{j, k}-y_{j, \ell}\right| \quad k, \ell=0, \ldots, N_{j}^{h}, j \in J .
\end{aligned}
$$

\section{A finite difference scheme for the stationary MFG system}

In this section we introduce the approximation scheme for the system (1.1). For simplicity, we consider a network $\Gamma$ without boundary; appropriate boundary condition can be inserted in the scheme in a straightforward way. At the internal grid points we consider the finite difference system

$$
\begin{cases}-\nu_{j}\left(D_{h}^{2} U\right)_{j, k}+g\left(x_{j, k},\left[D_{h} U\right]_{j, k}\right)+\Lambda=\left(V_{h}[M]\right)_{j, k}, & k=1, \ldots, N_{j}^{h}-1, j \in J \\ \nu_{j}\left(D_{h}^{2} M\right)_{j, k}+\mathcal{B}^{h}(U, M)_{j, k}=0, & k=1, \ldots, N_{j}^{h}-1, j \in J \\ M \in \mathcal{K}_{h}, \quad(U, 1)_{2}=0, & \end{cases}
$$

where $U, M$ are grid functions and $\Lambda \in \mathbb{R}$. The transport operator $\mathcal{B}^{h}$ is defined for $j \in J$ and $k=1$ by

$$
\begin{aligned}
\mathcal{B}^{h}(U, M)_{j, k}= & \frac{1}{h_{j}}\left[M_{j, k} \frac{\partial g}{\partial q_{1}}\left(x_{j, k},\left[D_{h} U\right]_{j, k}\right)+\right. \\
& \left.M_{j, k+1} \frac{\partial g}{\partial q_{2}}\left(x_{j, k+1},\left[D_{h} U\right]_{j, k+1}\right)-M_{j, k} \frac{\partial g}{\partial q_{2}}\left(x_{j, k},\left[D_{h} U\right]_{j, k}\right)\right] ;
\end{aligned}
$$

for $k=2, \ldots, N_{j}^{h}-2$ by

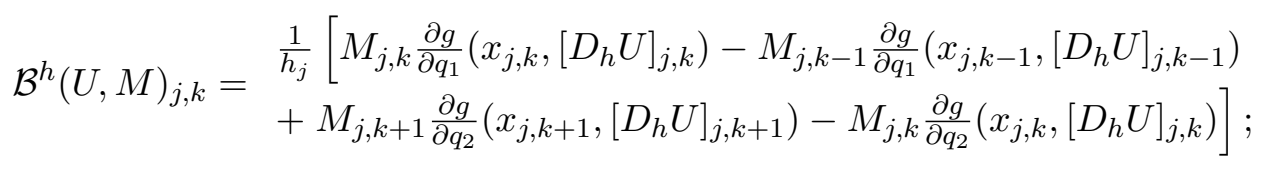

for $k=N_{j}^{h}-1$ by

$$
\mathcal{B}^{h}(U, M)_{j, k}=\begin{aligned}
& \frac{1}{h_{j}}\left[M_{j, k} \frac{\partial g}{\partial q_{1}}\left(x_{j, k},\left[D_{h} U\right]_{j, k}\right)-M_{j, k-1} \frac{\partial g}{\partial q_{1}}\left(x_{j, k-1},\left[D_{h} U\right]_{j, k-1}\right)-\right. \\
& \left.M_{j, k} \frac{\partial g}{\partial q_{2}}\left(x_{j, k},\left[D_{h} U\right]_{j, k}\right)\right] .
\end{aligned}
$$

We discuss now the transition conditions at the vertices, see (1.2). We discretize the Kirchhoff condition for the function $u$ via a $1^{\text {st }}$ order approximation of the derivative and we impose the continuity at the vertices

$$
\begin{cases}\mathcal{S}^{h}\left(U, V_{h}[M]-\Lambda\right)_{i}=0, & i \in I, \\ U \text { continuous at } v_{i}, & i \in I,\end{cases}
$$


where for grid functions $U, V$, the operator $\mathcal{S}^{h}: \mathcal{V} \rightarrow \mathbb{R}$ is defined by

$$
\mathcal{S}^{h}(U, V)_{i}=\sum_{j \in \operatorname{Inc}_{i}^{+}}\left[\nu_{j}\left(D^{+} U\right)_{j, 0}+\frac{h_{j}}{2} V_{j, 0}\right]-\sum_{j \in \operatorname{Inc}_{i}^{-}}\left[\nu_{j}\left(D^{+} U\right)_{j, N_{j}^{h}-1}-\frac{h_{j}}{2} V_{j, N_{j}^{h}}\right] .
$$

To discretize the transition condition for $m$ we consider a $1^{s t}$ order approximation of the derivative (the continuity of $M$ at the vertices is included in the definition of $\mathcal{K}_{h}$ )

$$
\mathcal{T}^{h}(M, U)_{i}=0 \quad i \in I,
$$

where for grid functions $U, M$, the operator $\mathcal{T}: \mathcal{V} \rightarrow \mathbb{R}$ is defined by

$$
\begin{aligned}
\mathcal{T}^{h}(M, U)_{i} & =\sum_{j \in \operatorname{Inc}_{i}^{+}}\left[\nu_{j}\left(D^{+} M\right)_{j, 0}+M_{j, 1} \frac{\partial g}{\partial q_{2}}\left(x_{j, 1},\left[D_{h} U\right]_{j, 1}\right)\right] \\
& -\sum_{j \in \operatorname{Inc}_{i}^{-}}\left[\nu_{j}\left(D^{+} M\right)_{j, N_{j}^{h}-1}+M_{j, N_{j}^{h}-1} \frac{\partial g}{\partial q_{1}}\left(x_{j, N_{j}^{h}-1},\left[D_{h} U\right]_{j, N_{j}^{h}-1}\right)\right]=0 .
\end{aligned}
$$

Remark 3.1 For the discretization of the differential equations in (1.1) inside the edge, we follow the same approach in [1, 2] and we refer to these papers for motivations and explanations. We just recall that the transport operator $\mathcal{B}^{h}$ comes from the discretization of the quantity

$$
\int_{e_{j}} m H_{p}(x, \partial u) \partial w d x
$$

for a test function $w$, which is connected with the weak formulation of the Fokker-Planck equation on the network.

For the the approximation of the transition conditions in $(1.2)$, we use a standard $1^{\text {st }}$ order discretization of the normal derivative of $u$ and $m$ with the sign depending if the vertex corresponds to either the initial point or the terminal one in the parametrization of the edge. The flux term in the Kirchhoff condition for $m$ is approximated in a upwind fashion depending always on the orientation of the edge. Finally the additional term $\frac{h_{j}}{2}\left(\left(V_{h}[M]\right)-\Lambda\right)$ in (3.2), which vanishes for $h \rightarrow 0$, is necessary to obtain the identity (3.22) which plays a key role in the uniqueness and convergence results.

Note that at a vertex $v_{i}$, we have respectively \# $\left(\right.$ Inc $\left._{i}\right)$ values $U_{j}$ and \# $\left(\right.$ Inc $\left._{i}\right)$ values $M_{j}$, corresponding to the restrictions of these functions to the incident edges $e_{j}, j \in$ Inc $_{i}$. Since (3.2) and (3.4) gives \#(Inc $\left.c_{i}\right)$ linear conditions, the value of $U$ and $M$ at $v_{i}$ is univocally determined.

Summarizing the approximation scheme for the stationary problem (1.1) is given by the (3.1)-(3.5). In the next subsections we study existence, uniqueness and convergence of the scheme.

\subsection{Existence}

We prove existence of a solution to (3.1)- 3.5 by a fixed point argument. We preliminarily need to prove existence, uniqueness and regularity for the first 
equation in (3.1) with transition conditions (3.2), see Lemma 3.4. This result is obtained, as in the continuous case, by approximating the limit ergodic problem (3.15) with the sequence of problems (3.6), which contains a zero order term $\rho U^{\rho}$, and passing to the limit for $\rho \rightarrow 0$. For this we need to estimate, uniformly in $\rho$, the discrete gradient of $U^{\rho}$ (see Lemma 3.14).

Lemma 3.1 Let $V: \mathcal{G}_{h} \rightarrow \mathbb{R}$ be a continuous grid function and assume that $g$ satisfies (G1)-(G3). For $\rho>0$, there is a unique solution to the problem

$$
\begin{cases}-\nu_{j}\left(D_{h}^{2} U^{\rho}\right)_{j, k}+g\left(x_{j, k},\left[D_{h} U^{\rho}\right]_{j, k}\right)+\rho U_{j, k}^{\rho}=V_{j, k}, & k=1, \ldots, N_{j}^{h}-1, j \in J \\ \mathcal{S}^{h}\left(U^{\rho}, V-\rho U^{\rho}\right)_{i}=0, & i \in I ; \\ U^{\rho} \text { continuous at } v_{i}, & i \in I .\end{cases}
$$

Proof To prove the existence we show that the map $\mathcal{F}: \mathbb{R}^{N^{h}} \rightarrow \mathbb{R}^{N^{h}}$ defined by

$$
\mathcal{F}(U)=\left\{\begin{array}{l}
\frac{1}{\rho}\left(\nu_{j}\left(D_{h}^{2} U\right)_{j, k}-g\left(x_{j, k},\left[D_{h} U\right]_{j, k}\right)+V_{j, k}\right), \quad j \in J, k=1, \ldots, N_{j}^{h}-1 \\
\frac{1}{\rho h_{v_{i}}} \mathcal{S}^{h}(U, V)_{i}, \quad i \in I
\end{array}\right.
$$

(where $h_{v_{i}}$ as in (2.2)) admits a fixed point.

Set $r=\left(\max _{j, k}\left|H\left(x_{j, k}, 0\right)\right|+\|V\|_{\infty}\right) / \rho$. By the regularity of $g$ the map $\mathcal{F}$ is continuous from $B_{r}=\left\{U \in \mathbb{R}^{N^{h}}:\|U\|_{\infty} \leq r\right\}$ to $\mathbb{R}^{N^{h}}$. Assume that $U \in \partial B_{r}$, hence $\max _{j \in J, k=0, \ldots, N_{j}^{h}}\left|U_{j, k}\right|=r$. Consider first the case $U_{j, k}=r$ for some $j \in J, k \in$ $\left\{1, \ldots, N_{j}^{h}-1\right\}$. Since $\left(D_{h}^{2} U\right)_{j, k} \leq 0, D^{+} U_{j, k} \leq 0$ and $D^{+} U_{j, k-1} \geq 0$, by the monotonicity and the consistency of $g$ we get

$$
\nu_{j}\left(D_{h}^{2} U\right)_{j, k}-g\left(x_{j, k},\left[D_{h} U\right]_{j, k}\right) \leq-H\left(x_{j, k}, 0\right)
$$

and therefore

$$
\mathcal{F}(U)_{j, k} \leq \frac{1}{\rho}\left(-H\left(x_{j, k}, 0\right)+V_{j, k}\right) \leq r .
$$

Hence $\mathcal{F}(U)_{j, k} \leq U_{j, k}$ and $\mathcal{F}(U)_{j, k} \neq \mu U_{j, k}$ if $\mu>1$.

Now assume that there exists $i \in I$ such that $U_{i}=r$ for some $i \in I$ ( $U_{i}$ is the common value of $U_{j, k}$ at $\left.v_{i}\right)$ then $\left(D^{+} U\right)_{j, 0} \leq 0$ if $v_{i}=\pi_{j}(0),\left(D^{+} U\right)_{j, N_{j}^{h}-1} \geq 0$ if $v_{i}=\pi_{j}\left(N_{j}^{h}\right)$ and therefore

$$
\mathcal{F}(U)_{i} \leq \frac{2}{\rho h_{v_{i}}}\left(\sum_{j \in \operatorname{Inc}_{i}^{+}} \frac{h_{j}}{2} V_{j, 0}+\sum_{j \in \operatorname{Inc}_{i}^{-}} \frac{h_{j}}{2} V_{j, N_{j}^{h}}\right) \leq r .
$$

Hence $\mathcal{F}(U)_{i} \leq U_{i}$ and $\mathcal{F}(U)_{i} \neq \mu U_{i}$ if $\mu>1$. Arguing in a similar way if either $U_{j, k}=-r$ or $U_{i}=-r$, we have that $\mathcal{F}(U) \neq \mu U$ for all $\mu>1$ and $U \in \partial B_{r}$. Hence by the LeraySchauder fixed point theorem there exists $U^{\rho} \in B_{r}$ such that $\mathcal{F}\left(U^{\rho}\right)=U^{\rho}$ and therefore a solution of (3.6). We also have the estimate

$$
\left\|U^{\rho}\right\|_{\infty} \leq \frac{1}{\rho}\left(\max _{j, k}\left|H\left(x_{j, k}, 0\right)\right|+\|V\|_{\infty}\right) .
$$

We prove uniqueness of the solution to (3.6). Let $U^{1}, U^{2}$ be two solutions of (3.6) and assume by contradiction that $\max _{j, k}\left(U_{j, k}^{1}-U_{j, k}^{2}\right)=\delta>0$. Consider first the case that 
there exists $\bar{j} \in J, \bar{k} \in\left\{1, \ldots, N_{j}^{h}-1\right\}$ such that $U_{\bar{j}, \bar{k}}^{1}-U_{\bar{j}, \bar{k}}^{2}=\delta$. Subtracting the equations satisfied by $U^{1}$ and $U^{2}$, we get

$$
-\nu_{j}\left(D_{h}^{2}\left(U^{1}-U^{2}\right)\right)_{\bar{j}, \bar{k}}+g\left(x_{\bar{j}, \bar{k}},\left[D_{h} U^{1}\right]_{j, k}\right)-g\left(x_{\bar{j}, \bar{k}},\left[D_{h} U^{2}\right]_{j, k}\right)+\rho\left(U^{1}-U^{2}\right)_{\bar{j}, \bar{k}}=0 .
$$

Since $(\bar{j}, \bar{k})$ is a maximum point for $U^{1}-U^{2}$, by the monotonicity of $g$, we get

$$
\rho \delta=\rho\left(U^{1}-U^{2}\right)_{\bar{j}, \bar{k}} \leq 0
$$

and therefore a contradiction. If there exists $i \in I$ such that $U_{i}^{1}-U_{i}^{2}=\delta$, then subtracting the transition conditions satisfied by $U^{1}$ and $U^{2}$, we get

$$
\begin{aligned}
0 & =\sum_{j \in \operatorname{Inc}_{i}^{+}}\left(\nu_{j}\left(D^{+}\left(U^{1}-U^{2}\right)\right)_{j, 0}-\frac{h_{j}}{2}\left(\rho\left(U^{1}-U^{2}\right)_{j, 0}\right)\right. \\
& -\sum_{j \in \operatorname{Inc}_{i}^{-}}\left(\nu_{j}\left(D^{+}\left(U^{1}-U^{2}\right)\right)_{j, N_{j}^{h}-1}-\frac{h_{j}}{2} \rho\left(U^{1}-U^{2}\right)_{j, N_{j}^{h}}\right) \leq-\frac{\delta \rho}{2} \sum_{j \in \operatorname{Inc}_{i}} h_{j}
\end{aligned}
$$

and therefore also in this case a contradiction. We conclude that $U^{1} \leq U^{2}$ and we prove in a similar way that $U^{2} \leq U^{1}$.

In the next lemma, we get an a priori bound for the gradient of the solution to the discrete Hamilton-Jacobi-Bellman equation by assuming that the function is bounded. It is important for the analysis of the convergence of the scheme that all the bounds are uniform in $h$.

Lemma 3.2 Let $\tilde{V}: \mathcal{G}_{h} \rightarrow \mathbb{R}$ be a continuous grid function and assume that $g$ satisfies (G1)-(G4). Let $U^{h}$ be a solution of the problem

$$
\begin{cases}-\nu_{j}\left(D_{h}^{2} U\right)_{j, k}+g\left(x_{j, k},\left[D_{h} U\right]_{j, k}\right)=\tilde{V}_{j, k}, & k=1, \ldots, N_{j}^{h}-1, j \in J \\ \mathcal{S}^{h}(U, \tilde{V})_{i}=0 & i \in I ; \\ U \text { continuous at } v_{i}, & i \in I,\end{cases}
$$

and assume that

$$
\left\|U^{h}\right\|_{\infty} \leq C_{0}
$$

with $C_{0}$ independent of $h$. Then

$$
\left\|D_{h} U^{h}\right\|_{\infty}:=\max _{j \in J} \max _{k=0, \ldots, N_{j}^{h}-1}\left|\left(D^{+} U^{h}\right)_{j, k}\right| \leq C
$$

where $C$ depends on $C_{0},\|\tilde{V}\|_{\infty}$, but not on $h$.

Proof We first prove that $D^{+} U^{h}$ is bounded at the vertices. Assume by contradiction that for some $i \in I$

$$
\max \left\{\max _{j \in \operatorname{Inc}_{i}^{+}}\left|\left(D^{+} U^{h}\right)_{j, 0}\right|, \max _{j \in \operatorname{Inc}_{i}^{-}}\left|\left(D^{+} U^{h}\right)_{j, N_{j}^{h}-1}\right|\right\} \rightarrow+\infty \quad \text { for }|h| \rightarrow 0 .
$$


Because of the transition condition in (3.8), it is not restrictive to assume that, up to a subsequence,

$$
\max \left\{\max _{j \in \operatorname{Inc}_{i}^{+}}\left\{\left(D^{+} U^{h}\right)_{j, 0}\right\}, \max _{j \in \operatorname{Inc}_{i}^{-}}\left\{-\left(D^{+} U^{h}\right)_{j, N_{j}^{h}-1}\right\}\right\} \rightarrow+\infty \quad \text { for }|h| \rightarrow 0 .
$$

Hence we assume that there exists $j \in \mathrm{Inc}_{i}^{+}$such that $D^{+} U_{j, 0}^{h} \rightarrow+\infty$ for $h_{j} \rightarrow 0$ (we proceed in a similar way if there exists $j \in \operatorname{Inc}_{i}^{-}$such that $-D^{+} U_{j, N_{j}^{h}-1}^{h} \rightarrow+\infty$ ).

Let $h_{0}$ be such that for $h_{j}<h_{0}$

$$
D^{+} U_{j, 0}^{h} \geq \frac{1}{\alpha}\left(C+\|\tilde{V}\|_{\infty}\right)+\frac{4 C_{0}}{l_{j}}
$$

where $C$ as in (G4), $C_{0}$ as in $(3.9)$. Since $\left(D_{h}^{2} U^{h}\right)_{j, 1}=\left(D^{+} U_{j, 1}^{h}-D^{+} U_{j, 0}^{h}\right) / h$ we have

$$
\begin{gathered}
\frac{\nu_{j}}{h_{j}} D^{+} U_{j, 1}^{h}=\frac{\nu_{j}}{h_{j}} D^{+} U_{j, 0}^{h}+g\left(x_{j, 1},\left[D_{h} U^{h}\right]_{j, 1}\right)-\tilde{V}_{j, k} \geq \\
\frac{\nu_{j}}{h_{j}} D^{+} U_{j, 0}^{h}+\alpha\left|D^{+} U_{j, 0}^{h}\right|^{\gamma}-C-\|\tilde{V}\|_{\infty} \geq \frac{\nu_{j}}{h_{j}} D^{+} U_{j, 0}^{h}
\end{gathered}
$$

and therefore $D^{+} U_{j, 1}^{h} \geq D^{+} U_{j, 0}^{h}$. Iterating the previous inequality, we get

$$
D^{+} U_{j, k+1}^{h} \geq D^{+} U_{j, k}^{h} \quad \text { for } k=0, \ldots, N_{j}^{h}-1 .
$$

For $L \leq N_{j}^{h}-1$, we have

$$
\left\{\begin{array}{l}
U_{j, 1}^{h}=U_{j, 0}^{h}+h_{j} D^{+} U_{j, 0}^{h}, \\
U_{j, 2}^{h}=U_{j, 1}^{h}+h_{j} D^{+} U_{j, 1}^{h}=U_{j, 0}^{h}+h_{j}\left(D^{+} U_{j, 0}^{h}+D^{+} U_{j, 1}^{h}\right), \\
\vdots \\
U_{j, L}^{h}=U_{j, 0}^{h}+h_{j} \sum_{k=0}^{L-1} D^{+} U_{j, k}^{h} .
\end{array}\right.
$$

If $L h_{j}>l_{j} / 2$, by (3.11) we get

$$
U_{j, L}^{h} \geq U_{j, 0}^{h}+L h_{j} D^{+} U_{j, 0}^{h} \geq U_{j, 0}^{h}+L h_{j} \frac{4 C_{0}}{l_{j}}>C_{0}
$$

and therefore a contradiction to 3.9 . .

We show that $D^{+} U^{h}$ is bounded also inside $\Gamma$. Assume by contradiction that there exists $j \in J, k_{h} \in\left\{1, \ldots, N_{j}^{h}-2\right\}$ such that, up to a subsequence,

$$
\left|D^{+} U_{j, k_{h}}^{h}\right| \rightarrow+\infty \quad \text { for } h \rightarrow 0 .
$$

By compactness, $x_{j, k_{h}} \rightarrow x_{0} \in e_{j}$ for $h \rightarrow 0$. We set $y_{0}=\pi_{j}^{-1}\left(x_{0}\right) \in\left[0, l_{j}\right]$ and we first consider the case $y_{0} \in\left(0, l_{j}\right)$. If $D^{+} U_{j, k_{h}}^{h} \rightarrow+\infty$ for $h_{j} \rightarrow 0$, let $h_{0}$ be such that for $h_{j} \leq h_{0}$

$$
D^{+} U_{j, k_{h}}^{h} \geq \frac{1}{\alpha}\left(C+\|\tilde{V}\|_{\infty}\right)+\frac{4 C_{0}}{l_{j}-y_{0}} .
$$

Arguing as in (3.10), we have

$$
D^{+} U_{j, k_{h}+l}^{h} \geq D^{+} U_{j, k_{h}+l-1}^{h} \quad \text { for } l=1, \ldots, N_{j}^{h}-k_{h} .
$$


For $L h_{j}>\left(l_{j}-y_{0}\right) / 2$ we get

$$
D^{+} U_{j, k_{h}+L}^{h}=U_{j, k_{h}}^{h}+h_{j} \sum_{l=0}^{L-1} D^{+} U_{j, k_{h}+l}^{h} \geq-C_{0}+L h \frac{4 C_{0}}{l_{j}-y_{0}}>C_{0}
$$

and therefore a contradiction to $(3.9)$.

We now consider the case $y_{0} \in\left(0, l_{j}\right)$ and $D^{+} U_{j, k_{h}}^{h} \rightarrow-\infty$ for $h \rightarrow 0$. Let $h_{0}$ be such that for $h_{j} \leq h_{0}$

$$
D^{+} U_{j, k_{h}}^{h} \leq-\frac{1}{\alpha}\left(C+\|\tilde{V}\|_{\infty}\right)-\frac{4 C_{0}}{y_{0}}
$$

We have

$$
\begin{aligned}
& \frac{\nu_{j}}{h_{j}} D^{+} U_{j, k_{h}}^{h}=\frac{\nu_{j}}{h_{j}} D^{+} U_{j, k_{h}-1}^{h}+g\left(x_{j, 1},\left[D_{h} U^{h}\right]_{j, k_{h}}\right)-\tilde{V}_{j, k_{h}}^{h} \geq \\
& \frac{\nu_{j}}{h_{j}} D^{+} U_{j, k_{h}-1}^{h}+\alpha\left|D^{+} U_{j, k_{h}}^{h}\right|^{\gamma}-C-\|\tilde{V}\|_{\infty} \geq \frac{\nu_{j}}{h_{j}} D^{+} U_{j, k_{h}-1}^{h}
\end{aligned}
$$

and iterating

$$
D^{+} U_{j, k_{h}-l}^{h} \geq D^{+} U_{j, k_{h}-l-1}^{h} \quad \text { for } l=0, \ldots, k_{h}-1 .
$$

For $L \leq k_{h}-1$, we have

$$
\left\{\begin{array}{l}
U_{j, k_{h}-1}^{h}=U_{j, k_{h}}^{h}-h D^{+} U_{j, k_{h}-1}^{h} \\
\vdots \\
U_{j, k_{h}-L}^{h}=U_{j, k_{h}}^{h}-h \sum_{l=1}^{L} D^{+} U_{j, k_{h}-l}^{h}
\end{array}\right.
$$

Hence if $L h_{j}>y_{0} / 2$, by 3.13 we get

$$
U_{j, k_{h}-L}^{h} \geq U_{j, k_{h}}^{h}-h_{j} \sum_{l=1}^{L} D^{+} U_{j, k_{h}-l}^{h} \geq U_{j, k_{h}}^{h}-L h_{j} D^{+} U_{j, k_{h}}^{h} \geq-C_{0}+L h_{j} \frac{4 C_{0}}{y_{0}}>C_{0}
$$

and therefore a contradiction to 3.9 ).

In case $y_{0}=\pi_{j}^{-1}\left(x_{0}\right)$ is equal either to 0 or to $l_{j}$ and $\left|D^{+} U_{j, k_{h}}^{h}\right| \rightarrow+\infty$, it is easy to adapt the previous arguments to obtain again a contradiction to 3.9 .

Lemma 3.3 Let $U^{\rho}$ be the solution of $(3.6)$, then

$$
\left\|D^{+} U^{\rho}\right\|_{\infty} \leq C_{2}
$$

for a constant $C_{2}$ independent of $\rho$ and $h$.

Proof Fix an arbitrary node $x_{\bar{j}, \bar{k}} \in \Gamma$ and set $W^{\rho}=U^{\rho}-U_{\bar{j}, \bar{k}}^{\rho}$. Adapting to the case of the networks the argument in [2, Prop.2], it is possible to show that $W^{\rho}$ is bounded, uniformly in $\rho$. Since $W^{\rho}$ is a solution of (3.8) with $\tilde{V}=V-\rho U^{\rho}$ and by (3.7) $\tilde{V}$ is bounded, uniformly in $\rho$ and $h$, we can apply Lemma 3.2 to get a bound on $\left\|D W^{\rho}\right\|_{\infty}$ and therefore on $\left\|D U^{\rho}\right\|$ uniform in $\rho$ and $h$. 
Lemma 3.4 Let $V: \mathcal{G}_{h} \rightarrow \mathbb{R}$ be a continuous grid function and assume that $g$ satisfies (G1)-(G4). Then there exists a unique couple $(U, \Lambda)$, where $U: \mathcal{G}_{h} \rightarrow \mathbb{R}$ and $\Lambda \in \mathbb{R}$, solution of the problem

$$
\begin{cases}-\nu_{j}\left(D_{h}^{2} U\right)_{j, k}+g\left(x_{j, k},\left[D_{h} U\right]_{j, k}\right)+\Lambda=V_{j, k}, & k=1, \ldots, N_{j}^{h}-1, j \in J \\ \mathcal{S}^{h}(U, V-\Lambda)_{i}=0, & i \in I ; \\ U \text { continuous at } v_{i}, & i \in I \\ (U, 1)_{2}=0 . & \end{cases}
$$

Moreover

$$
|\Lambda| \leq C_{1}, \quad\left\|D_{h} U\right\|_{\infty} \leq C_{2}
$$

for some constants $C_{1}, C_{2}$ independent of $h$.

Proof We prove existence by passing to the limit in the ergodic approximation (3.6). By (3.7)

$$
\left\|\rho U^{\rho}\right\|_{\infty} \leq C_{1}
$$

for any $\rho>0$ where $C_{1}$ is independent of $\rho$. By (3.7) and (3.14), up to a subsequence, $U^{\rho}-\left(U^{\rho}, 1\right)_{2}$ converges to a function $U: \mathcal{G}_{h} \rightarrow \mathbb{R}$ such that $(U, 1)_{2}=0$ and $\rho U_{j, k}^{\rho}$ converges to $\Lambda \in \mathbb{R}$ (independent of $(j, k)$ ). Moreover the couple $(U, \Lambda)$ satisfies $(3.15)$ and the bounds in (3.16).

The uniqueness of the couple $(U, \Lambda)$ can be proved by an argument similar to the one for the uniqueness of (3.6).

Remark 3.2 Note that the dependence of the bounds in (3.16) on the function $V_{j, k}$ is only by means of $\|V\|_{\infty}$. This is crucial for the proof of the next theorem.

Theorem 3.1 If $g$ satisfies (G1)-(G4), V satisfies (V1), then the problem (3.1)-(3.5) has at least a solution $(U, M, \Lambda)$. Moreover

$$
|\Lambda| \leq C_{1}, \quad\|U\|_{\infty}+\left\|D_{h} U\right\|_{\infty} \leq C_{2}
$$

for some constants $C_{1}, C_{2}$ independent of $h$.

Proof We define a map $\Phi$ which associates to $M \in \mathcal{K}_{h}$ the solution $(U, \Lambda)$ of the problem (3.15) with $V_{j, k}=\left(V_{h}[M]\right)_{j, k}$. By Lemma 3.4 the map $\Phi$ is well defined.

We show that $\Phi$ is continuous. Let $M^{s} \in \mathcal{K}_{h}$ be such that $M^{s} \rightarrow M \in \mathcal{K}_{h}$ as $s \rightarrow \infty$, hence by (V1), $V_{h}\left[M^{s}\right] \rightarrow V_{h}[M]$ as $s \rightarrow \infty$. Let $\left(U^{s}, \Lambda^{s}\right)$ be the sequence of solutions of (3.15) with $V=V_{h}\left[M^{s}\right]$. By (3.16) the sequences $\Lambda^{s}$ and $\left\|U^{s}\right\|_{\infty}$ are bounded and therefore, up to a subsequence, converge to $\Lambda \in \mathbb{R}$ and, respectively, to a grid function $U$. It is immediate that $(U, \Lambda)$ is a solution of 3.15 with $V=V_{h}[M]$. By the uniqueness of the solution to (3.15), it follows that all the sequence $\left(\Lambda^{s}, U^{s}\right)$ converges to $(\Lambda, U)$ and therefore the continuity of the map $\Phi$ and the estimate (3.17).

We define a map $\Psi$ which associates to $M \in \mathcal{K}_{h}$ the solution $\bar{M}$ of linear problem

$$
\left\{\begin{array}{l}
\mu \bar{M}_{j, k}-\nu_{j}\left(D_{h}^{2} \bar{M}\right)_{j, k}-\mathcal{B}^{h}(U, \bar{M})_{j, k}=\mu M_{j, k} \quad j \in J, k=1, \ldots, N_{j}^{h}-1 \\
\mu \bar{M}_{i}-\sum_{j \in \operatorname{Inc}_{i}^{+}} \frac{2}{h_{j}}\left[\nu_{j}\left(D^{+} \bar{M}\right)_{j, 0}+\bar{M}_{j, 1} \frac{\partial g}{\partial q_{2}}\left(x_{j, 1},\left[D_{h} U\right]_{j, 1}\right)\right]+ \\
\sum_{j \in \operatorname{Inc}_{i}^{-}} \frac{2}{h_{j}}\left[\nu_{j}\left(D^{+} \bar{M}\right)_{j, N_{j}^{h}-1}-\bar{M}_{j, N_{j}^{h}-1} \frac{\partial g}{\partial q_{1}}\left(x_{j, N_{j}^{h}-1},\left[D_{h} U\right]_{j, N_{j}^{h}-1}\right)\right]=\mu M_{i} \quad i \in I
\end{array}\right.
$$


where $\mu>0$ and $(U, \Lambda)=\Phi(M)$. We rewrite the previous problem as

$$
\mu \bar{M}+\mathcal{A} \bar{M}=\mu M
$$

where $\mathcal{A}$ is $N^{h} \times N^{h}$ matrix. By the monotonicity and the regularity of $g$, for $\mu$ sufficiently large the matrix $\mu I+\mathcal{A}$ is a non singular $M$-matrix and is therefore invertible. It follows that for any $M \in \mathcal{K}_{h},(3.18$ admits a solution $\bar{M}$ and by $M$-matrix property $\bar{M} \geq 0$ since $M \geq 0$. We prove that $(\bar{M}, 1)_{2}=1$. First observe that if $W, Z: \mathcal{G}_{h} \rightarrow \mathbb{R}$, then

$$
\begin{aligned}
\sum_{j \in J} \sum_{k=1}^{N_{j}^{h}-1} \nu_{j}\left(D_{h}^{2} W\right)_{j, k} Z_{j, k}=-\sum_{j \in J} \sum_{k=1}^{N_{j}^{h}-2} \nu_{j}\left(D^{+} W\right)_{j, k}\left(D^{+} Z\right)_{j, k} \\
\quad-\sum_{i \in I} \sum_{j \in \mathrm{Inc}_{i}^{+}} \frac{\nu_{j}}{h_{j}} Z_{j, 1}\left(D^{+} W\right)_{j, 0}+\sum_{i \in I} \sum_{j \in \mathrm{Inc}_{i}^{-}} \frac{\nu_{j}}{h_{j}} Z_{j, N_{j-1}^{h}}\left(D^{+} W\right)_{j, N_{j}^{h}-1}
\end{aligned}
$$

and

$$
\begin{aligned}
\sum_{j \in J} \sum_{k=1}^{N_{j}^{h}-1} \mathcal{B}^{h}(U, W)_{j, k} Z_{j, k}=-\sum_{j \in J} \sum_{k=1}^{N_{j}^{h}-1} W_{j, k}\left[D_{h} Z\right]_{j, k} \cdot \nabla_{q} g\left(x_{j, k},\left[D_{h} U\right]_{j, k}\right) \\
-\sum_{i \in I}\left(\sum_{j \in \operatorname{Inc}_{i}^{+}} \frac{1}{h_{j}}\left[W_{j, 1} Z_{j, 0} \frac{\partial g}{\partial q_{2}}\left(x_{j, 1},\left[D_{h} U\right]_{j, 1}\right)\right]\right. \\
\left.-\sum_{j \in \operatorname{Inc}_{i}^{-}} \frac{1}{h_{j}}\left[W_{j, N_{j}^{h}-1} Z_{j, N_{j}^{h}} \frac{\partial g}{\partial q_{1}}\left(x_{j, N_{j}^{h}-1},\left[D_{h} U\right]_{j, N_{j}^{h}-1}\right)\right]\right)
\end{aligned}
$$

If $W=\bar{M}, Z \equiv 1$, by $(3.19)-(3.20)$ we get

$$
\begin{aligned}
& \sum_{j \in J} \sum_{k=1}^{N_{j}^{h}-1}(\mathcal{A} \bar{M})_{j, k}=\sum_{i \in I} \sum_{j \in \operatorname{Inc}_{i}^{+}} \frac{1}{h_{j}}\left[\nu_{j}\left(D^{+} \bar{M}\right)_{j, 0}+\bar{M}_{j, 1} \frac{\partial g}{\partial q_{2}}\left(x_{j, 1},\left[D_{h} U\right]_{j, 1}\right)\right] \\
& -\sum_{i \in I} \sum_{j \in \mathrm{Inc}_{i}^{-}} \frac{1}{h_{j}}\left[\nu_{j}\left(D^{+} \bar{M}\right)_{j, N_{j}^{h}-1}-\bar{M}_{j, N_{j}^{h}-1} \frac{\partial g}{\partial q_{1}}\left(x_{j, N_{j}^{h}-1},\left[D_{h} U\right]_{j, N_{j}^{h}-1}\right)\right]
\end{aligned}
$$

Hence by the definition of $\mathcal{A}$ at the vertices we have

$$
\sum_{j \in J} \sum_{k=1}^{N_{j}^{h}-1}(\mathcal{A} \bar{M})_{j, k}=\frac{\mu}{2} \sum_{i \in I}\left(\sum_{j \in \operatorname{Inc}_{i}^{+}}(\bar{M}-M)_{j, 0}+\sum_{j \in \operatorname{Inc}_{i}^{-}}(\bar{M}-M)_{j, N_{j}^{h}}\right) .
$$

Therefore

$$
\sum_{j \in J} \sum_{k=1}^{N_{j}^{h}-1} h_{j}\left[\mu \bar{M}_{j, k}+(\mathcal{A} \bar{M})_{j, k}\right]=\mu \sum_{j \in J} \sum_{k=1}^{N_{j}^{h}-1} h_{j} M_{j, k}
$$

which implies $(\bar{M}, 1)_{2}=(M, 1)_{2}=1$ and therefore $\bar{M} \in \mathcal{K}_{h}$.

Hence $\Psi$ maps $\mathcal{K}_{h}$ into $\mathcal{K}_{h}$. From the boundedness and continuity of $\Phi$ and the regularity of $g, \Psi$ is continuous. By the Brouwer's fixed point theorem it follows that $\Psi$ admits a fixed point $M$ which is a solution of (3.1)- (3.5). 


\subsection{Uniqueness}

We first prove a fundamental identity which plays a crucial role in uniqueness and convergence of the scheme (compare with [3, (3.20)].

Lemma 3.5 Let $A, B: \mathcal{G}_{h} \rightarrow \mathbb{R}$ be two grid functions, $(U, M, \Lambda)$ a solution of (3.1)-(3.5) and $(\bar{U}, \bar{M}, \bar{\Lambda})$ a solution of

$$
\begin{cases}-\nu_{j}\left(D_{h}^{2} \bar{U}\right)_{j, k}+g\left(x_{j, k},\left[D_{h} \bar{U}\right]_{j, k}\right)+\bar{\Lambda}=\left(V_{h}[\bar{M}]\right)_{j, k}+A_{j, k}, & k=1, \ldots, N_{j}^{h}-1, j \in J \\ \nu_{j}\left(D_{h}^{2} \bar{M}\right)_{j, k}+\mathcal{B}^{h}(\bar{U}, \bar{M})_{j, k}=B_{j, k}, & k=1, \ldots, N_{j}^{h}-1, j \in J \\ \mathcal{S}^{h}\left(\bar{U}, V_{h}[\bar{M}]-\Lambda\right)_{i}=\sum_{j \in I n c_{i}^{+}} \frac{h_{j}}{2} A_{j, 0}+\sum_{j \in I n c_{i}^{-}} \frac{h_{j}}{2} A_{j, N_{j}^{h}}, & i \in I \\ \mathcal{T}^{h}(\bar{M}, \bar{U})_{i}=\sum_{j \in \operatorname{Inc}_{i}^{+}} \frac{h_{j}}{2} B_{j, 0}+\sum_{j \in \operatorname{Inc}_{i}^{-}} \frac{h_{j}}{2} B_{j, N_{j}^{h}}, & i \in I \\ \bar{U} \text { continuous at } v_{i}, & i \in I \\ \bar{M} \in \mathcal{K}_{h}, \quad(\bar{U}, 1)_{2}=0 . & \end{cases}
$$

Then

$\mathcal{R}^{h}(M, U, \bar{U})+\mathcal{R}^{h}(\bar{M}, \bar{U}, U)+\left(V_{h}[M]-V_{h}[\bar{M}], M-\bar{M}\right)_{2}=(A, M-\bar{M})_{2}+(B, U-\bar{U})_{2}$

where

$$
\begin{aligned}
\mathcal{R}^{h}(M, U, \bar{U})=\sum_{j \in J} \sum_{k=1}^{N_{j}^{h}-1} h_{j} & M_{j, k}\left[g\left(x_{j, k},\left[D_{h} \bar{U}\right]_{j, k}\right)-g\left(x_{j, k},\left[D_{h} U\right]_{j, k}\right)\right. \\
- & {\left.\left[D_{h}(\bar{U}-U)\right]_{k, j} \cdot \nabla_{q} g\left(x_{j, k},\left[D_{h} U\right]_{j, k}\right)\right] }
\end{aligned}
$$

Proof Let $(U, M, \Lambda)$ and $(\bar{U}, \bar{M}, \bar{\Lambda})$ be as in the statement. Subtracting the equations for $U$ and $\bar{U}$, multiplying the resulting equation by $h_{j}(M-\bar{M})_{j, k}$ and summing over $j \in J$, $k=1, \ldots, N_{j}^{h}-1$, we get

$$
\begin{gathered}
\sum_{j \in J} \sum_{k=1}^{N_{j}^{h}-1} h_{j}(M-\bar{M})_{j, k}\left[-\nu_{j} D_{h}^{2}(U-\bar{U})_{j, k}+g\left(x_{j, k},\left[D_{h} U\right]_{j, k}\right)-g\left(x_{j, k},\left[D_{h} \bar{U}\right]_{j, k}\right)+\right. \\
\left.(\Lambda-\bar{\Lambda})-\left(V_{h}[M]-V_{h}[\bar{M}]\right)_{j, k}\right]=\sum_{j \in J} \sum_{k=1}^{N_{j}^{h}-1} h_{j}(M-\bar{M})_{j, k} A_{j, k} .
\end{gathered}
$$

Subtracting the equations for $M$ and $\bar{M}$, multiplying the resulting equation by $h_{j}(U-\bar{U})_{j, k}$ and summing over $j \in J, k=1, \ldots, N_{j}^{h}-1$, we get

$$
\begin{gathered}
\sum_{j \in J} \sum_{k=1}^{N_{j}^{h}-1} h_{j}(U-\bar{U})_{j, k}\left[\nu_{j} D_{h}^{2}(M-\bar{M})_{j, k}+\mathcal{B}^{h}(U, M)_{j, k}-\mathcal{B}^{h}(\bar{U}, \bar{M})_{j, k}\right]= \\
\sum_{j \in J} \sum_{k=1}^{N_{j}^{h}-1} h_{j}(U-\bar{U})_{j, k} B_{j, k} .
\end{gathered}
$$


We have the identity

$$
\begin{array}{r}
-\sum_{j \in J} \sum_{k=1}^{N_{j}^{h}-1} \nu_{j}(U-\bar{U})_{j, k} D_{h}^{2}(M-\bar{M})_{j, k}=\sum_{j \in J} \sum_{k=1}^{N_{j}^{h}-1}-\nu_{j}(M-\bar{M})_{j, k} D_{h}^{2}(U-\bar{U})_{j, k} \\
+\sum_{j \in \operatorname{Inc}_{i}^{+}} \frac{\nu_{j}}{h_{j}}\left[(M-\bar{M})_{j, 0}\left(D^{+}(U-\bar{U})\right)_{j, 0}-(U-\bar{U})_{j, 0}\left(D^{+}(M-\bar{M})\right)_{j, 0}\right] \\
+\sum_{j \in \operatorname{Inc}_{i}^{-}} \frac{\nu_{j}}{h_{j}}\left[(M-\bar{M})_{j, N_{j}^{h}}\left(D^{+}(U-\bar{U})\right)_{j, N_{j-1}^{h}}-(U-\bar{U})_{j, N_{j}^{h}}\left(D^{+}(M-\bar{M})\right)_{j, N_{j-1}^{h}}\right]
\end{array}
$$

and, respectively,

$$
\begin{aligned}
& \sum_{j \in J} \sum_{k=1}^{N_{j}^{h}-1} \mathcal{B}^{h}(U, M)_{j, k}(U-\bar{U})_{j, k}=-\sum_{j \in J}\left[\sum_{k=2}^{N_{j}^{h}-2} M_{j, k}\left[D_{h}(U-\bar{U})\right]_{j, k} \cdot \nabla_{q} g\left(x_{j, k},\left[D_{h} U\right]_{j, k}\right)\right. \\
& \left.-M_{j, 1} \frac{\partial g}{\partial q_{2}}\left(x_{j, 1},\left[D_{h} U\right]_{j, 1}\right)(\bar{U}-U)_{j, 0}+M_{j, N_{j}^{h}-1} \frac{\partial g}{\partial q_{1}}\left(x_{j, N_{j}^{h}-1},\left[D_{h} U\right]_{j, N_{j}^{h}-1}\right)(\bar{U}-U)_{j, N_{j}^{h}}\right] .
\end{aligned}
$$

In a similar way a corresponding equation for $\sum_{j \in J} \sum_{k=1}^{N_{j}^{h}-1} \mathcal{B}^{h}(\bar{U}, \bar{M})_{j, k}(U-\bar{U})_{j, k}$ is also obtained.

We now discuss the boundary terms in (3.25) and (3.26). By the transition conditions for $U$ and $\bar{U}$ and the continuity of $M$ at the vertices we have

$$
\begin{aligned}
& -\sum_{j \in \operatorname{Inc}_{i}^{+}} \frac{\nu_{j}}{h_{j}}(M-\bar{M})_{j, 0}\left(D^{+}(U-\bar{U})\right)_{j, 0}+\sum_{j \in \operatorname{Inc}_{i}^{-}} \frac{\nu_{j}}{h_{j}}(M-\bar{M})_{j, N_{j}^{h}}\left(D^{+}(U-\bar{U})\right)_{j, N_{j-1}^{h}} \\
& =\sum_{j \in \operatorname{Inc}_{i}^{+}} \frac{1}{2}(M-\bar{M})_{j, 0}\left[\left(V_{h}[M]-V_{h}[\bar{M}]\right)_{j, 0}-(\Lambda-\bar{\Lambda})-A_{j, 0}\right] \\
& +\sum_{j \in \operatorname{Inc}_{i}^{-}} \frac{1}{2}(M-\bar{M})_{j, N_{j}^{h}}\left[\left(V_{h}[M]-V_{h}[\bar{M}]\right)_{j, N_{j}^{h}}-(\Lambda-\bar{\Lambda})-A_{j, N_{j}^{h}}\right] .
\end{aligned}
$$

By transition conditions for $M$ and $\bar{M}$ we get

$$
\begin{aligned}
& \sum_{j \in \operatorname{Inc}_{i}^{+}} \frac{\nu_{j}}{h_{j}}(U-\bar{U})_{j, 0}\left(D^{+}(M-\bar{M})\right)_{j, 0}-\sum_{j \in \operatorname{Inc}_{i}^{-}} \frac{\nu_{j}}{h_{j}}(U-\bar{U})_{j, N_{j}^{h}}\left(D^{+}(M-\bar{M})\right)_{j, N_{j}^{h}-1} \\
& =-\sum_{j \in \operatorname{Inc}_{i}^{+}} \frac{(U-\bar{U})_{j, 0}}{h_{j}}\left[M_{j, 1} \frac{\partial g}{\partial q_{2}}\left(x_{j, 1},\left[D_{h} U\right]_{j, 1}\right)-\bar{M}_{j, 1} \frac{\partial g}{\partial q_{2}}\left(x_{j, 1},\left[D_{h} \bar{U}\right]_{j, 1}\right)\right] \\
& +\sum_{j \in \operatorname{Inc}_{i}^{-}} \frac{(U-\bar{U})_{j, N_{j}^{h}}}{h_{j}}\left[M_{j, N_{j}^{h}-1} \frac{\partial g}{\partial q_{1}}\left(x_{j, N_{j}^{h}-1},\left[D_{h} U\right]_{j, N_{j}^{h}-1}\right)\right. \\
& \left.-\bar{M}_{j, N_{j}^{h}-1} \frac{\partial g}{\partial q_{1}}\left(x_{j, N_{j}^{h}-1},\left[D_{h} \bar{U}\right]_{j, N_{j}^{h}-1}\right)\right]+\sum_{j \in \operatorname{Inc}_{i}^{+}} \frac{1}{2} B_{j, 0}(U-\bar{U})_{j, 0} \\
& +\sum_{j \in \operatorname{Inc}_{i}^{-}} \frac{1}{2} B_{j, N_{j}^{h}}(U-\bar{U})_{j, N_{j}^{h}} .
\end{aligned}
$$


Replacing (3.25)-(3.28) in (3.24) and adding the resulting equation to 3.23 we finally get (recall that $\left.(M, 1)_{2}=(M, 1)_{2}=1\right)$

$$
\begin{aligned}
\sum_{j \in J} \sum_{k=1}^{N_{j}^{h}-1} h_{j}[ & M_{j, k}\left(\begin{array}{c}
g\left(x_{j, k},\left[D_{h} \bar{U}\right]_{j, k}\right)-g\left(x_{j, k},\left[D_{h} U\right]_{j, k}\right) \\
-\left[D_{h}(\bar{U}-U)\right]_{k, j} \cdot \nabla_{q} g\left(x_{j, k},\left[D_{h} U\right]_{j, k}\right)
\end{array}\right) \\
& \left.+\bar{M}_{j, k}\left(\begin{array}{c}
g\left(x_{j, k},\left[D_{h} U\right]_{j, k}\right)-g\left(x_{j, k},\left[D_{h} \bar{U}\right]_{j, k}\right) \\
-\left[D_{h}(U-\bar{U})\right]_{k, j} \cdot \nabla_{q} g\left(x_{j, k},\left[D_{h} \bar{U}\right]_{j, k}\right.
\end{array}\right)\right] \\
& +\left(V_{h}[M]-V_{h}[\bar{M}], M-\bar{M}\right)_{2}=(A, M-\bar{M})_{2}+(B, U-\bar{U})_{2}
\end{aligned}
$$

which amounts to 3.22 .

Theorem 3.2 If $g$ satisfies (G1)-(G5) and the operator $V_{h}$ is strictly monotone, i.e.

$$
\left(V_{h}[M]-V_{h}[\bar{M}], M-\bar{M}\right)_{2} \leq 0 \Rightarrow M=\bar{M}
$$

then the problem (3.1)-(3.5) has at most one solution.

Proof Let $(U, M, \Lambda)$ and $(\bar{U}, \bar{M}, \bar{\Lambda})$ be two solutions of (3.1)-(3.5). By (3.22) with $A \equiv B \equiv 0$ we get

$$
\mathcal{R}^{h}(M, U, \bar{U})+\mathcal{R}^{h}(\bar{M}, \bar{U}, U)+\left(V_{h}[M]-V_{h}[\bar{M}], M-\bar{M}\right)_{2}=0 .
$$

By the convexity of $g$ and the monotonicity of $V$, we see that all the terms in the left hand side of the previous equality are positive and therefore must vanish. The strong monotonicity of $V$ implies that $M=\bar{M}$. Hence $(U, \Lambda),(\bar{U}, \bar{\Lambda})$ solve (3.15) with $V_{j, k}=$ $V_{h}[M]_{j, k}=V_{h}[\bar{M}]_{j, k}$ and by Lemma 3.4 we get $U=\bar{U}$ and $\Lambda=\bar{\Lambda}$.

\subsection{Convergence}

In this section we analyze the convergence of the scheme $3.1-3.5$ in the reference case

$$
H(x, p)=|p|^{\beta}+f(x)
$$

where $\beta \geq 2$ and $f: \Gamma \rightarrow \mathbb{R}$ is a continuous function. By [7, we know that in this case there exists a unique solution $(u, m, \lambda)$ to 1.1$)$ with $u \in C^{2, \alpha}(\Gamma), m \in C^{2}(\Gamma), m>0$, and $\lambda \in \mathbb{R}$.

We consider a numerical Hamiltonian of the form

$$
g(x, p)=G\left(p_{1}^{-}, p_{2}^{+}\right)+f(x)
$$

where $G\left(p_{1}, p_{2}\right)=\left(p_{1}^{2}+p_{2}^{2}\right)^{\beta / 2}$ and $p_{s}^{ \pm}$denote the positive and negative part of $p_{s}, s=1,2$. We observe that $g$ satisfies assumptions (G1)-(G5). We need an additional assumption: (V4) For any $m \in \mathcal{K}:=\left\{\mu \in C^{0, \alpha}(\Gamma): \int_{\Gamma} \mu d x=1\right\}, M \in \mathcal{K}^{h}$, denoted by $\mathcal{I}^{h}(M)$ the continuous piecewise linear reconstruction of $M \in \mathcal{K}^{h}$ on $\Gamma$, then

$$
\left\|V[m]-V_{h}[M]\right\|_{\infty} \leq \omega\left(\left\|m-\mathcal{I}^{h}(M)\right\|_{\infty}\right)
$$

where $\omega$ is a continuous, increasing function such that $\lim _{t \rightarrow 0^{+}} \omega(t)=0$. 
In the following we denote by $o(1)$ a generic grid function whose maximum norm tends to 0 as $|h| \rightarrow 0$. Given a solution $(u, m, \lambda)$ of (1.1), we define a grid function $u^{h}$ by

$$
\begin{aligned}
& u_{j, k}^{h}:=\frac{1}{h_{j}} \int_{\left|y-y_{j, k}\right| \leq h_{j} / 2} u_{j}(y) d y, \quad \text { if } j \in J, k=1, \ldots, N_{j}^{h}-1, \\
& u_{i}^{h}:=\sum_{j \in \operatorname{Inc}_{i}^{+}} \frac{2}{h_{j}} \int_{0 \leq y-y_{j, 0} \leq h_{j} / 2} u_{j}(y) d y+\sum_{j \in \operatorname{Inc}_{i}^{-}} \frac{2}{h_{j}} \int_{0 \leq y_{j, N_{j}^{h}}-y \leq h_{j} / 2} u_{j}(y) d y, \quad \text { if } i \in I .
\end{aligned}
$$

(note that $\left(u^{h}, 1\right)_{2}=0$ ). We define in a similar way the grid function $m^{h} \in \mathcal{K}_{h}$ and we also set $\lambda^{h}:=\lambda$. Observe that by $(\mathbf{V 4})$

$$
\lim _{h \rightarrow 0}\left\|V[m]-V_{h}\left[m^{h}\right]\right\|_{\infty}=0 .
$$

Hence by $(3.32)$ and the consistency assumption $(\mathbf{G 2}),\left(u^{h}, m^{h}, \lambda^{h}\right)$ is a solution of

$$
\begin{cases}-\nu_{j}\left(D_{h}^{2} u^{h}\right)_{j, k}+g\left(x_{j, k},\left[D_{h} u^{h}\right]_{j, k}\right)+\lambda^{h}=\left(V_{h}\left[m^{h}\right]\right)_{j, k}+A_{j, k}^{h}, & k=1, \ldots, N_{j}^{h}-1, j \in J \\ \nu_{j}\left(D_{h}^{2} m^{h}\right)_{j, k}+\mathcal{B}^{h}\left(u^{h}, m^{h}\right)_{j, k}=B_{j, k}^{h}, & k=1, \ldots, N_{j}^{h}-1, j \in J \\ m^{h} \in \mathcal{K}_{h}, \quad\left(u^{h}, 1\right)_{2}=0 & \end{cases}
$$

with the transition conditions

$$
\begin{cases}\mathcal{S}^{h}\left(u^{h}, V_{h}\left[m^{h}\right]-\lambda^{h}\right)_{i}=\sum_{j \in \operatorname{Inc}_{i}^{+}} \frac{h_{j}}{2} A_{j, 0}^{h}+\sum_{j \in \operatorname{Inc}_{i}^{-}} \frac{h_{j}}{2} A_{j, N_{j}^{h}}^{h}, & i \in I \\ \mathcal{T}^{h}\left(m^{h}, u^{h}\right)_{i}=\sum_{j \in \operatorname{Inc}_{i}^{+}} \frac{h_{j}}{2} B_{j, 0}^{h}+\sum_{j \in \operatorname{Inc}_{i}^{-}} \frac{h_{j}}{2} B_{j, N_{j}^{h}}^{h}, & i \in I \\ u^{h} \text { continuous at } v_{i}, & i \in I\end{cases}
$$

where $A^{h}, B^{h}$ are two grid functions such that

$$
\lim _{h \rightarrow 0}\left\|A^{h}\right\|_{\infty}=0, \quad \lim _{h \rightarrow 0}\left\|B^{h}\right\|_{\infty}=0 .
$$

We need some preliminary lemmas.

Lemma 3.6 Let $\beta \geq 2$,

i) For all $q, \tilde{q} \in \mathbb{R}^{2}$,

$$
g(x, \tilde{q})-g(x, q)-\nabla_{q} g(x, q) \cdot(\tilde{q}-q) \geq \frac{1}{\beta-1} \max \left(|p|^{\beta-2},|\tilde{p}|^{\beta-2}\right)|p-\tilde{p}|^{2}
$$

where $p=\left(q_{1}^{-}, q_{2}^{+}\right), \tilde{p}=\left(\tilde{q}_{1}^{-}, \tilde{q}_{2}^{+}\right)$.

ii) There exists a constant $C$ such that for all $q, \tilde{q}, r \in \mathbb{R}^{2}$ and $\eta>0$

$$
\left|\left(\nabla_{q} g(x, \tilde{q})-\nabla_{q} g(x, q)\right) \cdot r\right| \leq \max \left(|p|^{\beta-2},|\tilde{p}|^{\beta-2}\right)\left(\frac{C}{\eta}|p-\tilde{p}|^{2}+\eta|r|^{2}\right),
$$

where $p=\left(q_{1}^{-}, q_{2}^{+}\right), \tilde{p}=\left(\tilde{q}_{1}^{-}, \tilde{q}_{2}^{+}\right)$.

For the proof of the previous lemma, we refer to [3, Lemma 3.2]. 
Lemma 3.7 Let $\left(U^{h}, M^{h}, \Lambda^{h}\right)$ be a solution of (3.1)-(3.5) and $\left(u^{h}, m^{h}, \lambda^{h}\right)$ a solution of (3.33)-3.34 with $m^{h} \geq \delta>0$ for $h$ sufficiently small. Then

$$
\lim _{h \rightarrow 0} \sum_{j \in J} \sum_{k=1}^{N_{j}^{h}-1} h_{j}\left|\left[D^{+} U\right]_{j, k}-\left[D^{+} u^{h}\right]_{j, k}\right|^{\beta}=0
$$

Proof By the identity (3.22) with $(U, M, \Lambda)=\left(U^{h}, M^{h}, \Lambda^{h}\right)$ and $(\bar{U}, \bar{M}, \bar{\Lambda})=\left(u^{h}, m^{h}, \lambda^{h}\right)$ we get

$$
\begin{aligned}
\mathcal{R}^{h}\left(M^{h}, U^{h}, u^{h}\right)+\mathcal{R}^{h}\left(m^{h}, u^{h}, U^{h}\right)+\left(V_{h}\left[M^{h}\right]\right. & \left.-V_{h}\left[m^{h}\right], M^{h}-m^{h}\right)_{2} \\
& +\left(A^{h}, M^{h}-m^{h}\right)_{2}+\left(B^{h}, U^{h}-u^{h}\right)_{2}=0 .
\end{aligned}
$$

By (3.17) and the regularity of $u$ we get $\lim _{h \rightarrow 0}\left|\left(B^{h}, U^{h}-u^{h}\right)_{2}\right|=0$. By $m^{h}, M^{h} \in \mathcal{K}_{h}$ and the Cauchy-Schwarz also get $\lim _{h \rightarrow 0}\left|\left(A^{h}, M^{h}-m^{h}\right)_{2}\right|=0$. Hence by (3.36) we obtain

$$
\begin{aligned}
& \sum_{j \in J} \sum_{k=1}^{N_{j}^{h}} h_{j} m_{j, k}^{h} \max \left\{\left|P_{j, k}^{h}\right|^{\beta-2},\left|p_{j, k}^{h}\right|^{\beta-2}\right\}\left|P_{j, k}^{h}-p_{j, k}^{h}\right|^{2}=o(1), \\
& \sum_{j \in J} \sum_{k=1}^{N_{j}^{h}} h_{j} M_{j, k}^{h} \max \left\{\left|P_{j, k}^{h}\right|^{\beta-2},\left|p_{j, k}^{h}\right|^{\beta-2}\right\}\left|P_{j, k}^{h}-p_{j, k}^{h}\right|^{2}=o(1),
\end{aligned}
$$

where $P_{j, k}^{h}=\left(\left(D^{+} U^{h}\right)_{j, k}^{-},\left(D^{+} U^{h}\right)_{j, k-1}^{+}\right)$and $p_{j, k}^{h}=\left(\left(D^{+} u^{h}\right)_{j, k}^{-},\left(D^{+} u^{h}\right)_{j, k-1}^{+}\right)$. Since $m^{h}$ is strictly positive, by the first equation in (3.39) we get (3.38).

Theorem 3.3 Let $(u, m, \lambda)$ be the unique solution of (1.1) and $\left(U^{h}, M^{h}, \Lambda^{h}\right)$ the sequence of the solutions of the scheme (3.1)-(3.5). Then

$$
\lim _{|h| \rightarrow 0}\left\|U^{h}-u\right\|_{\infty}+\left\|M^{h}-m\right\|_{\infty}+\left|\Lambda^{h}-\lambda\right|=0 .
$$

Proof We set $E^{h}=M^{h}-m^{h}$. Subtracting the equations satisfied by $M^{h}$ and $m^{h}$ and multiplying the resulting equations for $h_{j} E_{j, k}^{h}$, we get

$$
\begin{aligned}
\sum_{j \in J} \sum_{k=1}^{N_{j}^{h}-1}\left[-\nu_{j}\left(D_{h}^{2} E^{h}\right)_{j, k}+\mathcal{B}^{h}\left(U^{h}, M^{h}\right)_{j, k}-\mathcal{B}^{h}\left(U^{h}, m^{h}\right)_{j, k}\right] h_{j} E_{j, k}^{h}= \\
-\sum_{j \in J} \sum_{k=1}^{N_{j}^{h}-1}\left[\mathcal{B}^{h}\left(u^{h}, m^{h}\right)_{j, k}-\mathcal{B}^{h}\left(U^{h}, m^{h}\right)_{j, k}+B_{j, k}^{h}\right] h_{j} E_{j, k}^{h} .
\end{aligned}
$$

By the transition conditions for $M^{h}$ and $m^{h}$ (recall that $M^{h}$ and, $m^{h}$ are continuous at 
the vertices)

$$
\begin{aligned}
& \sum_{i \in I}\left[\sum_{j \in \operatorname{Inc}_{i}^{+}} E_{j, 0}^{h}\left[\nu_{j}\left(D^{+} E^{h}\right)_{j, 0}+E_{j, 1}^{h} \frac{\partial g}{\partial q_{2}}\left(x_{j, 1}, D_{h} U^{h}\right]_{j, 1}\right]\right. \\
& \left.-\sum_{j \in \operatorname{Inc}_{i}^{-}} E_{j, N_{j}^{h}}^{h}\left[\nu_{j}\left(D^{+} E^{h}\right)_{j, N_{j}^{h}-1}+E_{j, N_{j}^{h}-1}^{h} \frac{\partial g}{\partial q_{1}}\left(x_{j, N_{j}^{h}-1},\left[D_{h} U^{h}\right]_{j, N_{j}^{h}-1}\right)\right]\right]= \\
& \sum_{i \in I}\left[\sum_{j \in \operatorname{Inc}_{i}^{+}} E_{j, 0}^{h}\left[m_{j, 1}^{h}\left(\frac{\partial g}{\partial q_{2}}\left(x_{j, 1},\left[D_{h} u^{h}\right]_{j, 1}\right)-\frac{\partial g}{\partial q_{2}}\left(x_{j, 1},\left[D_{h} U^{h}\right]_{j, 1}\right)\right)+\frac{h_{j}}{2} B_{j, 0}^{h}\right]\right. \\
& \left.-\sum_{j \in \operatorname{Inc}_{i}^{-}} E_{j, N_{j}^{h}}^{h}\left[m_{j, N_{j}^{h}-1}^{h}\left(\frac{\partial g}{\partial q_{1}}\left(x_{j, N_{j}^{h}-1},\left[D_{h} u^{h}\right]_{j, N_{j}^{h}-1}\right)-\frac{\partial g}{\partial q_{1}}\left(x_{j, N_{j}^{h}-1},\left[D_{h} U^{h}\right]_{j, N_{j}^{h}-1}\right)\right)+\frac{h_{j}}{2} B_{j, N_{j}^{h}}^{h}\right]\right] .
\end{aligned}
$$

Arguing as in (3.19)-(3.20), we have

$$
\begin{array}{r}
\sum_{j \in J} \sum_{k=1}^{N_{j}^{h}-1} \nu_{j}\left(D_{h}^{2} E^{h}\right)_{j, k} E_{j, k}^{h}=-\sum_{j \in J} \sum_{k=1}^{N_{j}^{h}-2} \nu_{j}\left|\left(D^{+} E^{h}\right)_{j, k}\right|^{2}-\sum_{i \in I}\left[\sum_{j \in \operatorname{Inc}_{i}^{+}} \nu_{j}\left|\left(D^{+} E^{h}\right)_{j, 0}\right|^{2}\right. \\
\left.+\sum_{j \in \operatorname{Inc}_{i}^{-}} \nu_{j}\left|\left(D^{+} E^{h}\right)_{j, N_{j}^{h}-1}\right|^{2}\right]+\sum_{i \in I}\left[-\sum_{j \in \operatorname{Inc}_{i}^{+}} \frac{\nu_{j}}{h_{j}} E_{j, 1}^{h}\left(D^{+} E^{h}\right)_{j, 0}+\sum_{j \in \operatorname{Inc}_{i}^{-}} \frac{\nu_{j}}{h_{j}} E_{j, N_{j}^{h}-1}^{h}\left(D^{+} E^{h}\right)_{j, N_{j}^{h}-1}\right] .
\end{array}
$$

Moreover

$$
\begin{aligned}
& \sum_{j \in J} \sum_{k=1}^{N_{j}^{h}-1}\left[\mathcal{B}^{h}\left(U^{h}, M^{h}\right)_{j, k}-\mathcal{B}^{h}\left(U^{h}, m^{h}\right)_{j, k}\right] E_{j, k}^{h}=-\sum_{j \in J} \sum_{k=1}^{N_{j}^{h}-1} E_{j, k}^{h}\left[D_{h} E^{h}\right]_{j, k} \cdot \nabla_{q} g\left(x_{j, k},\left[D_{h} U^{h}\right]_{j, k}\right) \\
- & \sum_{i \in I}\left[\sum_{j \in \operatorname{Inc}_{i}^{+}} \frac{1}{h_{j}} E_{j, 0}^{h} E_{j, 1}^{h} \frac{\partial g}{\partial q_{2}}\left(x_{j, 1},\left[D_{h} U^{h}\right]_{j, 1}\right)-\sum_{j \in \operatorname{Inc}_{i}^{-}} \frac{1}{h_{j}} E_{j, N_{j}^{h}}^{h} E_{j, N_{j}^{h}-1}^{h} \frac{\partial g}{\partial q_{1}}\left(x_{j, N_{j}^{h}-1},\left[D_{h} U^{h}\right]_{j, N_{j}^{h}-1}\right)\right] .
\end{aligned}
$$

and

$$
\begin{aligned}
\sum_{j \in J} \sum_{k=1}^{N_{j}^{h}-1} & {\left[\mathcal{B}^{h}\left(U^{h}, m^{h}\right)_{j, k}-\mathcal{B}^{h}\left(u^{h}, m^{h}\right)_{j, k}\right] E_{j, k}^{h} } \\
= & -\sum_{j \in J} \sum_{k=1}^{N_{j}^{h}-1} m_{j, k}^{h}\left[D_{h} E^{h}\right]_{j, k} \cdot\left(\nabla_{q} g\left(x_{j, k},\left[D_{h} u^{h}\right]_{j, k}\right)-\nabla_{q} g\left(x_{j, k},\left[D_{h} U^{h}\right]_{j, k}\right)\right) \\
& -\sum_{i \in I}\left[\sum_{j \in \operatorname{Inc}_{i}^{+}} \frac{1}{h_{j}} E_{j, 0}^{h} m_{j, 1}^{h}\left(\frac{\partial g}{\partial q_{2}}\left(x_{j, 1},\left[D_{h} u^{h}\right]_{j, 1}\right)-\frac{\partial g}{\partial q_{2}}\left(x_{j, 1},\left[D_{h} U^{h}\right]_{j, 1}\right)\right)\right. \\
& \left.-\sum_{j \in \operatorname{Inc}_{i}^{-}} \frac{1}{h_{j}} E_{j, N_{j}^{h}}^{h} m_{j, N_{j}^{h}-1}^{h}\left(\frac{\partial g}{\partial q_{1}}\left(x_{j, N_{j}^{h}-1},\left[D_{h} u^{h}\right]_{j, N_{j}^{h}-1}\right)-\frac{\partial g}{\partial q_{1}}\left(x_{j, N_{j}^{h}-1},\left[D_{h} U^{h}\right]_{j, N_{j}^{h}-1}\right)\right)\right] .
\end{aligned}
$$

Set

$$
\left(D^{h} E^{h}, D^{h} E^{h}\right)_{2}=\sum_{j \in J} \sum_{k=1}^{N_{j}^{h}-2}\left|\left(D^{+} E^{h}\right)_{j, k}\right|^{2}+\sum_{i \in I}\left[\sum_{j \in \operatorname{Inc}_{i}^{+}}\left|\left(D^{+} E^{h}\right)_{j, 0}\right|^{2}+\sum_{j \in \operatorname{Inc}_{i}^{-}}\left|\left(D^{+} E^{h}\right)_{j, N_{j}^{h}-1}\right|^{2}\right]
$$


Replacing the previous equalities in (3.41), using (3.42) and recalling the estimate $(3.16)$, we get

$$
\begin{aligned}
& \left(D^{h} E^{h}, D^{h} E^{h}\right)_{2} \leq-C\left[\sum_{j \in J} \sum_{k=1}^{N_{j}^{h}-1} h_{j} E_{j, k}^{h} A_{j, k}^{h}+h_{j} m_{j, k}^{h}\left[D_{h} E^{h}\right]_{j, k} \cdot\left(\nabla_{q} g\left(x_{j, k},\left[D_{h} u^{h}\right]_{j, k}\right)\right.\right. \\
& \left.\left.-\nabla_{q} g\left(x_{j, k},\left[D_{h} U^{h}\right]_{j, k}\right)\right)+\sum_{i \in I}\left(\sum_{j \in \operatorname{Inc}_{i}^{+}} h_{j} E_{j, 0}^{h} B_{j, 0}^{h}+\sum_{j \in \operatorname{Inc}_{i}^{-}} h_{j} E_{j, N_{j}^{h}}^{h} B_{j, N_{j}^{h}}^{h}\right)\right]
\end{aligned}
$$

with $C$ independent of $h$. By (3.35), we have

$$
\sum_{j \in J} h_{j} E_{j, k}^{h} A_{j, k}^{h}+\sum_{i \in I}\left[\sum_{j \in \operatorname{Inc}_{i}^{+}} h_{j} E_{j, 0}^{h} B_{j, 0}^{h}+\sum_{j \in \operatorname{Inc}_{i}^{-}} h_{j} E_{j, N_{j}^{h}}^{h} B_{j, N_{j}^{h}}^{h}\right] \leq o(1)\left(E_{h}, E_{h}\right)_{2} .
$$

Set $P^{h}=\left(\left(D^{+} U_{j, k}^{h}\right)^{-},\left(D^{+} U_{j, k-1}^{h}\right)^{+}\right), p^{h}=\left(\left(D^{+} u_{j, k}^{h}\right)^{-},\left(D^{+} u_{j, k-1}^{h}\right)^{+}\right)$. By (3.17), 3.37) for any $\eta>0$

$$
\begin{aligned}
& \left|m_{j, k}^{h}\left[D_{h} E^{h}\right]_{j, k} \cdot\left(\nabla_{q} g\left(x_{j, k},\left[D_{h} u^{h}\right]_{j, k}\right)-\nabla_{q} g\left(x_{j, k},\left[D_{h} U^{h}\right]_{j, k}\right)\right)\right| \\
& \leq m_{j, k}^{h} \max \left(\left|P_{j, k}^{h}\right|^{\beta-2},\left|p_{j, k}^{h}\right|^{\beta-2}\right)\left(\frac{C}{\eta}\left|P_{j, k}^{h}-p_{j, k}^{h}\right|^{2}+\eta\left|D^{h} E_{j, k}^{h}\right|^{2}\right) \\
& \leq m_{j, k}^{h}\left(\frac{C}{\eta}\left|\left[D^{h} U^{h}\right]_{j, k}-\left[D^{h} u^{h}\right]_{j, k}\right|^{\beta}+\eta\left|D^{h} E_{j, k}^{h}\right|^{2}\right) .
\end{aligned}
$$

Plugging the estimates (3.38), 3.44), (3.45) in 3.43), we finally get

$$
\left(E^{h}, E^{h}\right)_{2}+\left(D^{h} E^{h}, D^{h} E^{h}\right)_{2}=o(1) \quad \text { for }|h| \rightarrow 0 .
$$

Hence we get the convergence of $M^{h}$ to $m$ in $H^{1}(\Gamma)$ and uniform. By the convergence of $M^{h}$ to $m$ and (V4), we get $\lim _{h \rightarrow 0}\left\|V_{h}\left[M^{h}\right]-V_{h}\left[m^{h}\right]\right\|_{\infty}=0$. Hence $U^{h}$ and $u^{h}$ are solution of 3.15 with $\lambda=\Lambda^{h}, V_{j, k}=V_{h}\left[M^{h}\right]_{j, k}$ and respectively $\lambda=\lambda^{h}, V_{j, k}=V_{h}\left[M^{h}\right]_{j, k}+o(1)$. By a comparison principle for (3.15), we get $\left|\Lambda^{h}-\lambda^{h}\right| \leq o(1)$ and therefore

$$
\lim _{|h| \rightarrow 0}\left|\lambda-\Lambda^{h}\right|=0 .
$$

Let $\bar{u}^{h}$ be the continuous piecewise linear reconstruction of $U^{h}$ on $\Gamma$. By (3.17), $\bar{u}^{h} \rightarrow \bar{u}$ uniformly as $|h| \rightarrow 0$, up to a subsequence. By (3.38) and (3.46), $\bar{u}$ is a weak solution to (1.1). Therefore by the uniqueness of the solution to (1.1), we get the convergence of $U^{h}$ to $u$ in $H^{1}(\Gamma)$ and uniform.

\section{Numerical implementation and experiments}

This section is devoted to the implementation and test of a numerical solver for the stationary MFG system (3.1)-(3.5). In [2], the stationary MFG system on the torus is solved via the so called forward-forward long time approximation: for a given approximation step $h$, the approximate solution $\left(U_{h}, M_{h}, \Lambda_{h}\right)$ is obtained as the limit of $\left(U_{h}^{n}, M_{h}^{n}, U_{h}^{n} / n \Delta t\right)$ 
for $n \rightarrow \infty$, where $\left(U_{h}^{n}, M_{h}^{n}\right)$ is computed via discretization of the corresponding evolutive MFG system, implicit or explicit in time, up to time $T=n \Delta t$.

Here we propose a new approach which allows to compute the solution of the stationary MFG system directly, avoiding long time or small delta approximations. We collect all the unknowns $(U, M, \Lambda)$ in a single vector $X$ of length $2 N^{h}+1$ (with $N^{h}$ given by (2.2) ) and we recast the $2 N^{h}+2$ equations of the stationary MFG system as functions of $X$. Hence we get a nonlinear map $\mathcal{F}: \mathbb{R}^{2 N^{h}+1} \rightarrow \mathbb{R}^{2 N^{h}+2}$ defined by

$$
\mathcal{F}(X)= \begin{cases}-\nu_{j}\left(D_{h}^{2} U\right)_{j, k}+g\left(x_{j, k},\left[D_{h} U\right]_{j, k}\right)+\Lambda-\left(V_{h}[M]\right)_{j, k} & k=1, \ldots, N_{j}^{h}-1, j \in J \\ \nu_{j}\left(D_{h}^{2} M\right)_{j, k}+\mathcal{B}^{h}(U, M)_{j, k} & k=1, \ldots, N_{j}^{h}-1, j \in J \\ \mathcal{S}^{h}\left(U, V_{h}[M]-\Lambda\right)_{i} & i \in I \\ \mathcal{T}^{h}(M, U)_{i} & i \in I \\ (M, 1)_{2}-1 & \\ (U, 1)_{2} & \end{cases}
$$

and we look for $X^{\star} \in \mathbb{R}^{2 N^{h}+1}$ such that

$$
\mathcal{F}\left(X^{\star}\right)=0 \in \mathbb{R}^{2 N^{h}+2} .
$$

By Theorem 3.1 and Theorem 3.2 there exists a unique solution to 4.1), but the system is formally overdetermined, having $2 N^{h}+2$ equations in $2 N^{h}+1$ unknowns. This terminology normally applies to linear systems, but is commonly adopted also in the nonlinear case with a slight abuse of notation. Indeed, the solution is meant in the following nonlinear-least-squares sense:

$$
X^{\star}=\arg \min _{X} \frac{1}{2}\|\mathcal{F}(X)\|_{2}^{2} .
$$

To solve the above optimization problem, we employ the Gauss-Newton method, that we briefly recall here for completeness. We first denote the residual function by

$$
r(X)=\frac{1}{2}\|\mathcal{F}(X)\|_{2}^{2}=\frac{1}{2} \mathcal{F}(X)^{T} \mathcal{F}(X)
$$

and we consider the standard Newton method for approximating a critical point of $r$ :

$$
\mathcal{H}_{r}\left(X^{k}\right) \delta_{X}=-\nabla r\left(X^{k}\right), \quad X^{k+1}=X^{k}+\delta_{X}, \quad k \geq 0,
$$

where the gradient $\nabla r$ and the Hessian $\mathcal{H}_{r}$ are given by

$$
\nabla r(X)=J_{\mathcal{F}}(X)^{T} \mathcal{F}(X), \quad \mathcal{H}_{r}(X)=J_{\mathcal{F}}(X)^{T} J_{\mathcal{F}}(X)+\sum_{i=1}^{2 N^{h}+2} \frac{\partial^{2} \mathcal{F}_{i}}{\partial^{2} X}(X) \mathcal{F}_{i}(X),
$$

with

$$
\left(J_{\mathcal{F}}(X)\right)_{i, j}=\frac{\partial \mathcal{F}_{i}}{\partial X_{j}}(X), \quad\left(\frac{\partial^{2} \mathcal{F}_{i}}{\partial^{2} X}(X)\right)_{k, \ell}=\frac{\partial^{2} \mathcal{F}_{i}}{\partial X_{k} \partial X_{\ell}}(X) .
$$

Since we expect the residuals $\mathcal{F}_{i}\left(X^{k}\right)$ to be small for $X^{k}$ close enough to $X^{\star}$, it is reasonable to neglect the second derivatives in $\mathcal{H}_{r}$, using the approximation $\mathcal{H}_{r}(X) \simeq J_{\mathcal{F}}(X)^{T} J_{\mathcal{F}}(X)$. This yields the Gauss-Newton method:

$$
J_{\mathcal{F}}\left(X^{k}\right)^{T} J_{\mathcal{F}}\left(X^{k}\right) \delta_{X}=-J_{\mathcal{F}}\left(X^{k}\right)^{T} \mathcal{F}\left(X^{k}\right), \quad X^{k+1}=X^{k}+\delta_{X}, \quad k \geq 0,
$$


where the Jacobian $J_{\mathcal{F}}$ is well defined assuming that the numerical Hamiltonian $g$ is of class $C^{2}$ in the gradient variable and that the operator $V_{h}$ is of class $C^{1}$. Despite this method allows to employ only first order derivatives of $\mathcal{F}$, it is still not efficient from a numerical point of view. Indeed, once $J_{\mathcal{F}}$ at $X^{k}$ is computed, we also need to assemble the right hand side $J_{\mathcal{F}}^{T} \mathcal{F}$ and the matrix $J_{\mathcal{F}}^{T} J_{\mathcal{F}}$, typically squaring the condition number of the system. This can be avoided by simply realizing that the $k$-th iteration of the Gauss-Newton method is just the normal equation for the following linear-least-squares problem

$$
\min _{\delta_{X}} \frac{1}{2}\left\|J_{\mathcal{F}}\left(X^{k}\right) \delta_{X}+\mathcal{F}\left(X^{k}\right)\right\|_{2}^{2},
$$

which is in turn easily and efficiently solved by means of the $Q R$ factorization of $J_{\mathcal{F}}$. Indeed, let $m=2 N^{h}+2, n=2 N^{h}+1$ and suppose that $J_{\mathcal{F}}\left(X^{k}\right)=Q R$, where $Q$ is a $m \times m$ orthogonal matrix (i.e. $Q^{-1}=Q^{T}$ ) and $R$ is a $m \times n$ matrix of the form $R=\left(\begin{array}{c}R_{1} \\ 0\end{array}\right)$, with $R_{1}$ of size $n \times n$ and upper triangular. Writing $Q=\left(\begin{array}{ll}Q_{1} & Q_{2}\end{array}\right)$ with $Q_{1}$ of size $m \times n$ and $Q_{2}$ of size $m \times(m-n)$, we get

$$
\begin{gathered}
\left\|J_{\mathcal{F}}\left(X^{k}\right) \delta_{X}+\mathcal{F}\left(X^{k}\right)\right\|_{2}^{2}=\left\|Q^{T}\left(J_{\mathcal{F}}\left(X^{k}\right) \delta_{X}+\mathcal{F}\left(X^{k}\right)\right)\right\|_{2}^{2}=\left\|Q^{T} Q R \delta_{X}+Q^{T} \mathcal{F}\left(X^{k}\right)\right\|_{2}^{2}= \\
=\left\|\left(\begin{array}{c}
R_{1} \delta_{X} \\
0
\end{array}\right)+\left(\begin{array}{c}
Q_{1}^{T} \mathcal{F}\left(X^{k}\right) \\
Q_{2}^{T} \mathcal{F}\left(X^{k}\right)
\end{array}\right)\right\|_{2}^{2}=\left\|R_{1} \delta_{X}+Q_{1}^{T} \mathcal{F}\left(X^{k}\right)\right\|_{2}^{2}+\left\|Q_{2}^{T} \mathcal{F}\left(X^{k}\right)\right\|_{2}^{2}
\end{gathered}
$$

which is finally minimized by getting rid of the first of the two latter terms, i.e. solving the square triangular $n \times n$ linear system $R_{1} \delta_{X}=-Q_{1}^{T} \mathcal{F}\left(X^{k}\right)$ via back substitution.

Summarizing, we propose the following simple algorithm for the stationary MFG system:

Given a guess $X=\left(U^{0}, M^{0}, \Lambda^{0}\right)$, A tolerance $\varepsilon>0$ AND a DUMPing Parameter $0<\alpha \leq 1$,

REPEAT

- Assemble $\mathcal{F}(X)$ and $J_{\mathcal{F}}(X)$

- Solve the overdetermined linear system $J_{\mathcal{F}}(X) \delta_{X}=-\mathcal{F}(X)$ in the leastSQUARES SENSE 4.2 , USING THE $Q R$ FACTORIZATION OF $J_{\mathcal{F}}(X)$

- Update $X \leftarrow X+\alpha \delta_{X}$

UNTIL $\left\|\delta_{X}\right\|_{2}<\varepsilon$

The algorithm is implemented in C-language and employs the library SuiteSparseQR [11], which is designed to efficiently compute the $Q R$ factorization and the least-square solution to very large and sparse linear systems.

Some remarks are in order:

1) We always initialize the method by setting $U^{0} \equiv 0, \Lambda^{0}=0$ and $M^{0} \equiv 1 / L$, where $L=\sum_{j \in J} l_{j}$ is the total length of the network. In general there is no guarantee that the algorithm computes a minimum of 4.2 with zero residual, i.e. a solution of the stationary MFG system. Nevertheless, in all the tests performed, our algorithm seems to converge to a zero residual minimum independently on the initial guess.

2) As for the standard Newton method, it is known that also the Gauss-Newton method may not converge if the dumping parameter is set to $\alpha=1$. A fine tuning of $\alpha$ can 
be accomplished via some moderate time consuming line search technique, but for our purposes we simply checked that the fixed value $\alpha=0.9$ is sufficient in all the considered examples.

3) We never impose the constraint $M \geq 0$ in the computation. Surprisingly, our unconstrained optimization algorithm converges to a solution of the MFG system with non negative mass. We extensively checked this feature, also in the case of negative or changing sign initial guesses. Even if the mass can be negative in some intermediate iterations of the Gauss-Newton method, we always end up with a non negative mass in all the considered examples.

4) Our technique can be successfully applied also in the homogenization of HamiltonJacobi equations, e.g. for computing the effective Hamiltonian for some cell problems. Our preliminary tests using the nonlinear-least-squares approach are very promising, both in terms of accuracy and computational costs.

The previous points, in particular the convergence of the method, are still under investigation and will be addressed in a future work (see [6]).

We now set up the data for the numerical experiments. We consider a simple network in the plane with 2 vertices and 3 edges of unit length, as in Figure 2 a. For computational purposes the network is mapped in a topologically equivalent network, in which one vertex is located at the origin and the edges are delimited by the 3 rd roots of unity $\left(v_{j}=(\cos (2 \pi j / 3), \sin (2 \pi j / 3))\right.$, for $\left.j=0,1,2\right)$, as in Figure $2 \mathbf{b}$. Note that the boundary vertices, i.e. the vertices with a single incident edge, are identified and correspond to a single vertex on the network.

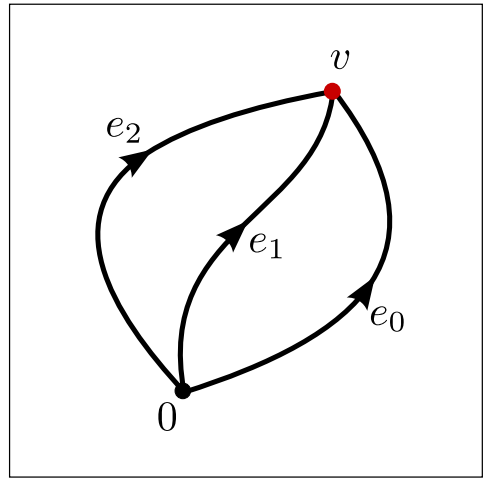

$(a)$

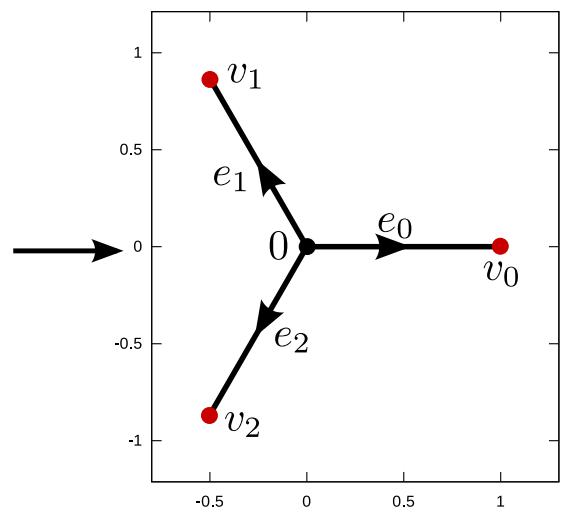

(b)

Figure 2: a network with 2 vertices and 3 edges (a) is mapped in an equivalent network with boundary vertices identified (b).

We assume that the numerical Hamiltonian has the form (3.31), with $\beta=2$ and $f(x)$ is such that, for $j=0,1,2$ and $x \in e_{j}$,

$$
f(x)=f_{j}(x):=s_{j}(1+\cos (2 \pi(t+1 / 2))), \quad x=t v_{j}, \quad t \in[0,1],
$$

where $s_{j} \in\{0,1\}$ is a switch parameter to activate/deactivate the corresponding cost on the edge $e_{j}$. If not differently specified, we discretize each edge by $N_{j}=250$ nodes, so that the resulting nonlinear system has dimension $1502 \times 1501$, and we choose a tolerance 
$\varepsilon=10^{-4}$ for the stopping criterion of the algorithm. We finally assume a uniform diffusion on the whole network, i.e. $\nu_{j} \equiv \nu$ for $j=0,1,2$ and $\nu>0$.

Here we are mainly interested in the qualitative behavior of the computed solutions, and we postpone at the end of the section some experimental analysis on the performance of the algorithm. Nevertheless, we remark that in all the following tests, the proposed method converges in about 10 iterations and the computational time is of the order of few seconds, even for larger grids.

All the tests were performed on a Lenovo Ultrabook X1 Carbon, using 1 CPU Intel Quad-Core i5-4300U 1.90Ghz with $8 \mathrm{~Gb}$ Ram, running under the Linux Slackware 14.1 operating system.

Test 1. We consider a local operator of the form $V_{h}[m]=m^{2}$, and we choose a diffusion coefficient $\nu=0.1$. Figure 3 shows the results corresponding to the activation of the cost $f$ on three, two or one edge, namely for $\left(s_{0}, s_{1}, s_{2}\right)=(1,1,1),\left(s_{0}, s_{1}, s_{2}\right)=(1,1,0)$ and $\left(s_{0}, s_{1}, s_{2}\right)=(1,0,0)$ respectively.
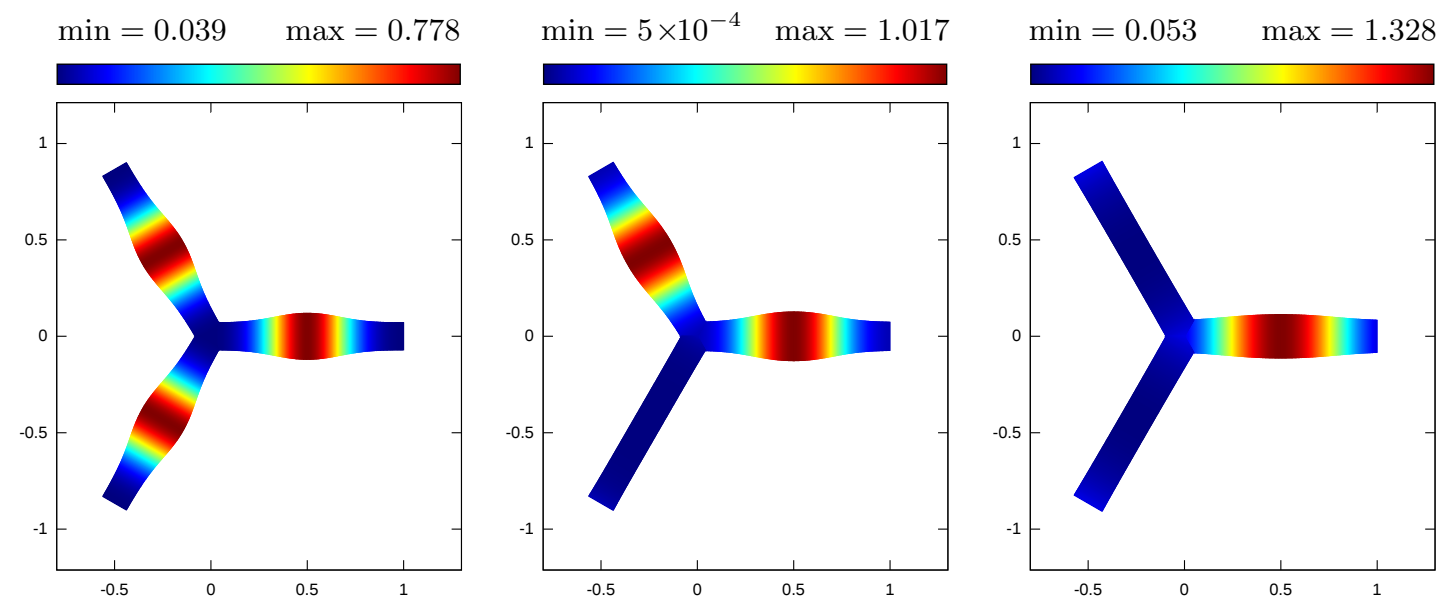

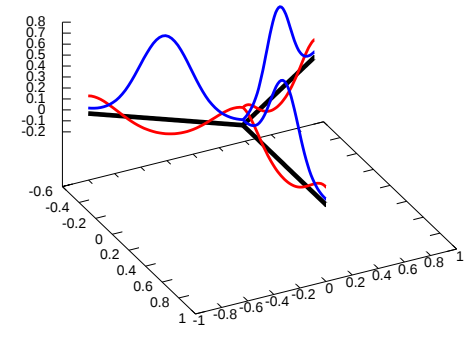

(a)

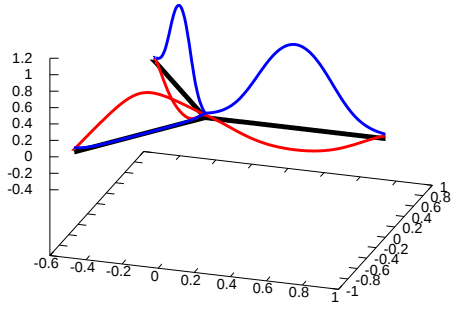

(b)

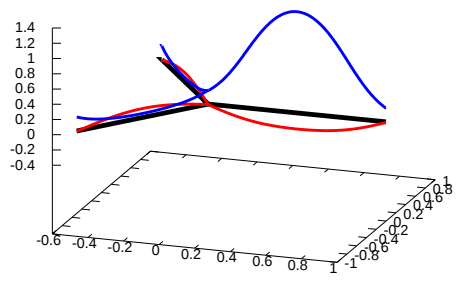

(c)

Figure 3: the case $V_{h}[m]=m^{2}$ and $\nu=0.1$, the cost $f$ is active on (a) three edges, (b) two edges, (c) one edge.

In the top panels we represent the mass $M$ using a color-map in which the blue and the red correspond respectively to the minimum and maximum values. Moreover, we represent the network as a fatten tube, whose cross sections have a size proportional to $M$ at the corresponding points. In the bottom panels we represent the network (in black) and both the mass $M$ (in blue) and the corresponding value function $U$ (in red). Since $V$ is 
increasing, it penalizes concentration of the mass. The cost $f$ has, if $s_{j} \neq 0$, a maximum in the center of the edge $e_{j}$. Hence, if $\nu$ is not to small, the agents should be well distributed on the network with a maximum of $m$ around the minima of the value functions $u$, i.e. in the center of the edges where the cost is active. In fact we observe this behavior in all the three examples.

Test 2. We are interested in the behavior of the solution as $\nu \rightarrow 0$, hence we choose the same parameters of the previous test, but with $\nu=10^{-4}$. In this respect, our method seems very robust and we can reach very small values of $\nu$ even for quite coarse grids. Figure 4 shows the corresponding results. In this case we see that the solution is not better than Lipschitz and the support of $D u$ and $m$ are disjoint, as in the Euclidean case (see [2, Test 2]).
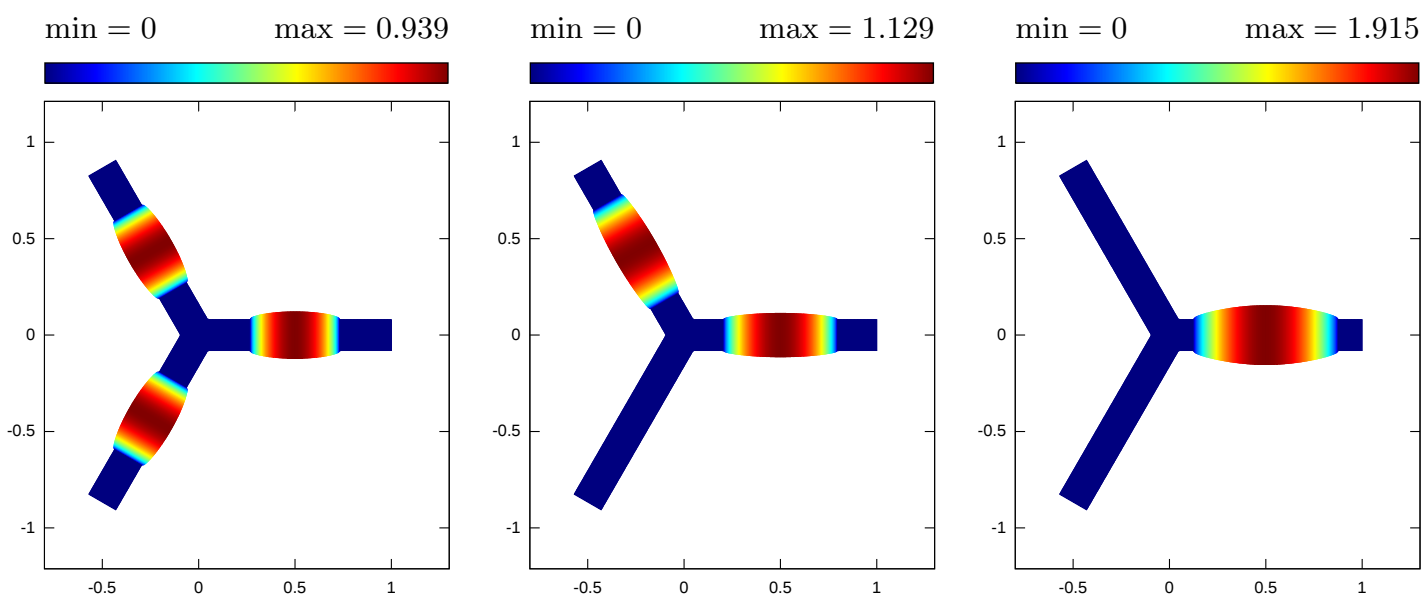

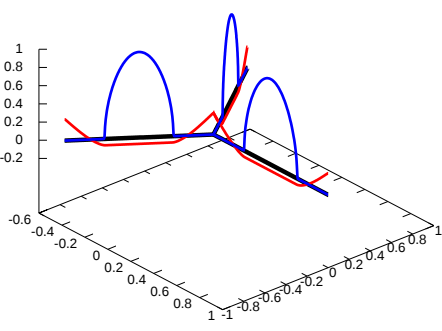

(a)

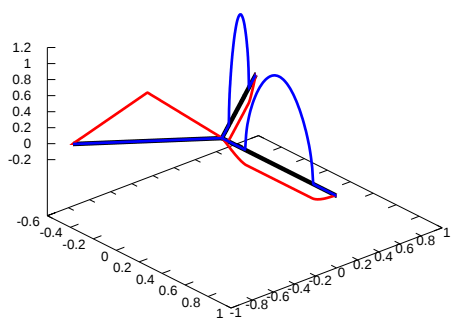

(b)

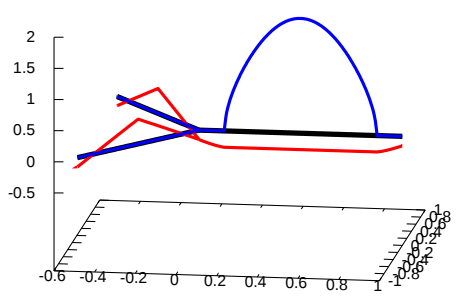

(c)

Figure 4: the case $V_{h}[m]=m^{2}$ and $\nu=10^{-4}$, the cost $f$ is active on (a) three edges, (b) two edges, (c) one edge.

Test 3. We set $V[m]=1-\frac{4}{\pi} \arctan (m)$, we consider both $\nu=0.1$ and $\nu=10^{-3}$ and the cost $f$ active on the whole network, i.e. $\left(s_{0}, s_{1}, s_{2}\right)=(1,1,1)$. Figure 5 shows the corresponding results. Since $V$ is decreasing, the agents want the share the same position and therefore tend to concentrate around the minima of the value function. Note that for $\nu$ small, the regularizing effect of the diffusion is small and $m$ is close to a sum of Dirac functions concentrated at the minima of $u$. In this case assumption $(3.29)$ is not satisfied and uniqueness of the solution may fail. 


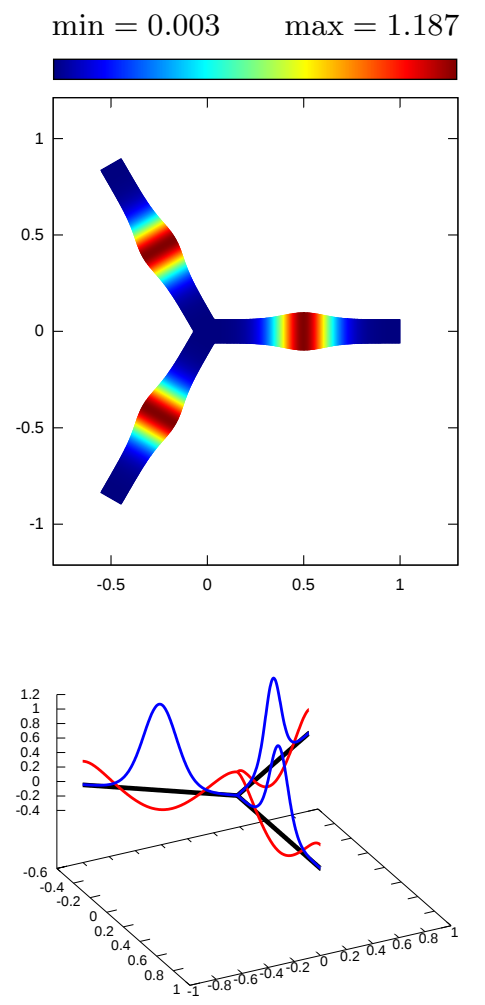

(a)

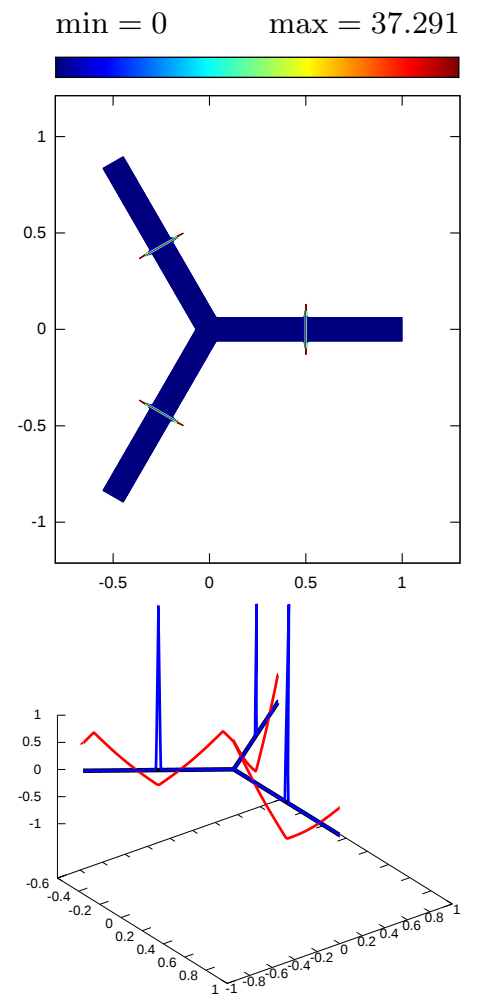

(b)

Figure 5: the case $V_{h}[m]=1-\frac{4}{\pi} \arctan (m)$ with (a) $\nu=0.1$ and (b) $\nu=10^{-3}$, the cost $f$ is active on the whole network.

Test 4. In this experiment, we show that the method can efficiently handle the computation on much more complicated structures. To this end, we consider the periodic network shown in Figure 6a. It is a self-similar set, in which the length of each edge scales with a factor $1 / 2$ when moving to adjacent edges. Starting from the longest edges, we stop at the second level of branching and we identify the extremal boundary vertices. Moreover, we choose the local operator $V_{h}[m]=m^{2}$, uniform diffusion coefficient $\nu=0.1$ and the cost $f$ as before, active on the whole network. In this example the players are distributed on all the edges with a scaling factor which depends on the length of the edge.

Performance and convergence. Here we present some results showing the convergence and performance of the proposed method, both in terms of accuracy and computational times. We consider the same setting of Test 1 , with the cost $f$ active on all the three edges of the network, i.e. $\left(s_{0}, s_{1}, s_{2}\right)=(1,1,1)$. Moreover, we choose the same number of discretization nodes for each edge, namely $N_{j}=N$ for $j=0,1,2$ and a variable $N$, so that the space step is $h=1 / N$ on the whole network. Note that, in the present case, the total number of degrees of freedom (dofs) of the problem is much more than $N$. Indeed, we have $N$ nodes for each of the three edges and for both $U$ and $M$, that is $\operatorname{dofs}=6 \mathrm{~N}$. Since the exact solution is unknown for this problem, we assume as correct the solution computed for $N=2000$, denoted by $\left(U^{e x}, M^{e x}, \Lambda^{e x}\right)$. Then we define 


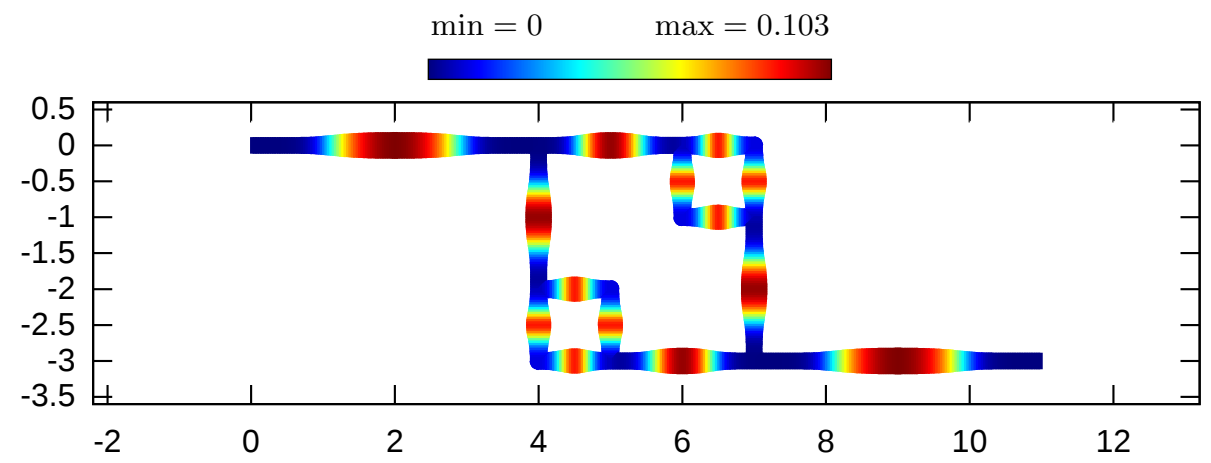

(a)

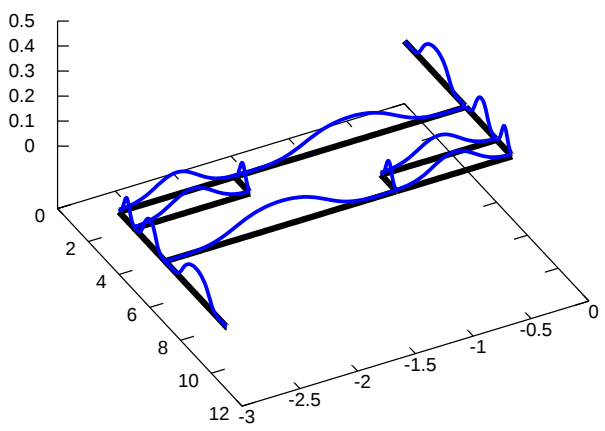

(b)

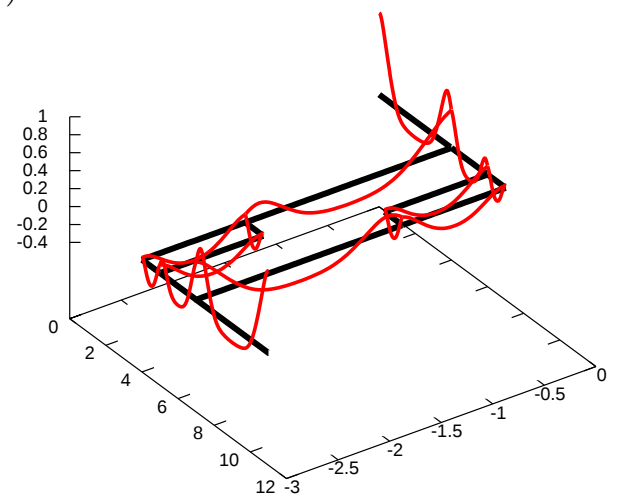

(c)

Figure 6: solution on a self-similar network, (a),(b) the mass $M$, (c) the value function $U$.

the error as

$$
E_{h}=\left\|U-U^{e x}\right\|_{1}+\left\|M-M^{e x}\right\|_{1}+\left|\Lambda-\Lambda^{e x}\right|,
$$

where the discrete $1-$ norm, for a generic vector $W$ with $3 N$ components, is computed as $\|W\|_{1}=h \sum_{k=1}^{3 N}\left|W_{k}\right|$ and the exact solution is projected on the corresponding grid via linear interpolation. Finally, we define the experimental order of convergence as $\boldsymbol{E} \boldsymbol{o c}\left(h_{1}, h_{2}\right)=\log \left(E_{h_{1}} / E_{h_{2}}\right) / \log \left(h_{1} / h_{2}\right)$ and we set $\varepsilon=10^{-8}$ for the stopping criterion of the algorithm.

In Figure 7a we show, for $N=1000$, the behavior of the computed $\Lambda$ as a function of the number of iterations. In this case $\Lambda^{e x}=-1.058687$, whereas $\Lambda=-1.058876$ is obtained after 20 iterations with $\left|\Lambda-\Lambda^{e x}\right|=0.000189$.

Similarly, in Figure $7 \mathbf{b}$ we plot the error $E_{h}$ for different space steps $h$, ranging from $10^{-2}$ to $10^{-3}$. This shows an experimental convergence at least of order 1.

Finally, in Table 1 we report all the results, including the error $\left|\Lambda-\Lambda^{e x}\right|$ related only to the approximation of the ergodic constant, the Eoc computed for successive space steps, the number of iterations and the corresponding computational times. We clearly see that, even for quite coarse grids, we get a reasonable approximation of the solution with a very low time consumption. 


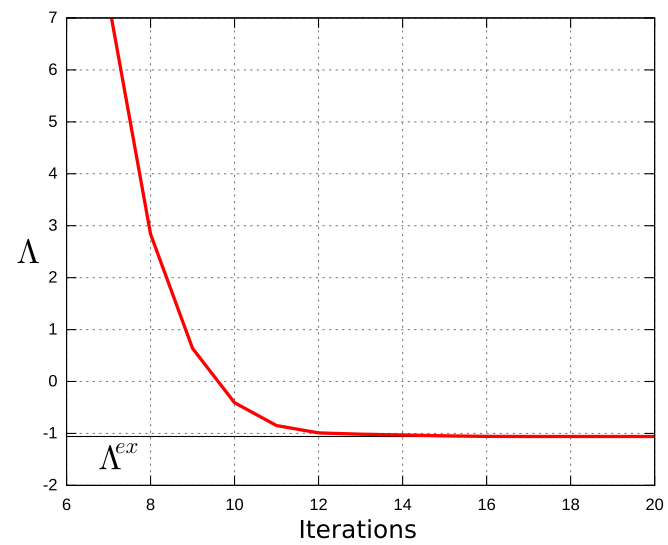

(a)

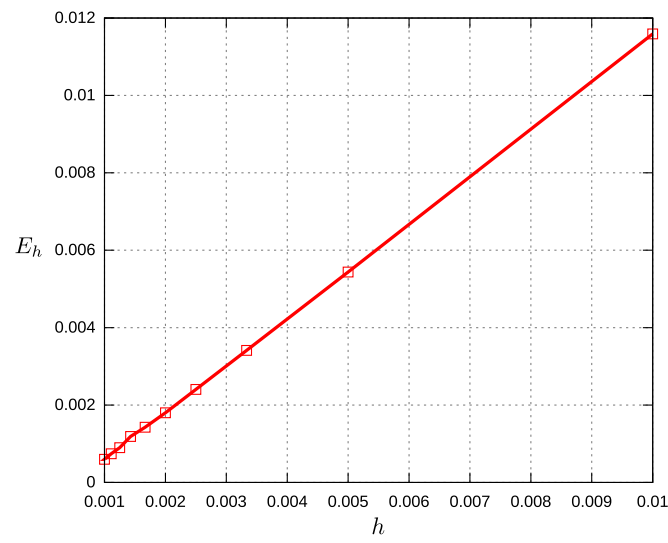

(b)

Figure 7: convergence, (a) $\Lambda$ vs number of iterations for $N=1000$, (b) error $E_{h}$ vs space step $h$.

\begin{tabular}{|c|c|c|c|c|c|c|}
\hline $\mathrm{N}$ & Dofs & Error $E_{h}$ & Error $\left|\Lambda-\Lambda^{\text {ex }}\right|$ & Iterations & Eoc & Cpu time \\
\hline 100 & 600 & 0.01159 & 0.003737 & 7 & - & 0.13 \\
\hline 200 & 1200 & 0.00544 & 0.001734 & 7 & 1.09 & 0.37 \\
\hline 400 & 2400 & 0.00241 & 0.000762 & 17 & 1.17 & 4.09 \\
\hline 800 & 4800 & 0.00091 & 0.000284 & 16 & 1.40 & 19.57 \\
\hline 1000 & 6000 & 0.00059 & 0.000189 & 20 & 1.94 & 47.94 \\
\hline
\end{tabular}

Table 1: Performance of the proposed method.

\section{References}

[1] Y. Achdou, Finite difference methods for mean field games. In Hamilton-Jacobi equations: approximations, numerical analysis and applications, 1-47, Lecture Notes in Math., 2074, Springer, Heidelberg, 2013.

[2] Y. Achdou and I. Capuzzo Dolcetta. Mean field games: numerical methods. SIAM J. Numer. Anal., 48(3) (2010), 1136-1162.

[3] Y. Achdou, F. Camilli, and I. Capuzzo Dolcetta. Mean field games: convergence of a finite difference method. SIAM J. Numer. Anal. 51 (2013), 2585-2612.

[4] Y. Achdou, J. Buera, J-M. Lasry, P-L. Lions and B. Moll, Partial differential equation models in macroeconomics. Phil. Trans. R. Soc. A, 372 (2014), no. 2078, 19 pp.

[5] G. Bretti, R. Natalini and B. Piccoli, Numerical approximations of a traffic flow model on networks. Netw. Heterog. Media 1 (2006), no. 1, 57-84.

[6] S. Cacace and F. Camilli, Effective Hamiltonian for Hamilton-Jacobi equations: yet another but efficient numerical method, in preparation.

[7] F. Camilli and C. Marchi, Stationary Mean Field Games on networks, arXiv:1505.04953, 2015. 
[8] F. Camilli, E. Carlini and C. Marchi, A model problem for Mean Field Games on networks, Discrete Contin. Dyn. Syst. 35 (2015), no. 9, 4173-4192.

[9] E. Carlini and F. Silva, A fully discrete Semi-Lagrangian scheme for a first order mean field games problem, SIAM J. Numer. Anal. 52 (2014), 45-67.

[10] C. D'Apice, R. Manzo and B. Piccoli, Numerical schemes for the optimal input flow of a supply chain. SIAM J. Numer. Anal. 51 (2013), no. 5, 2634-2650.

[11] T. Davis, SuiteSparse, http://faculty.cse.tamu.edu/davis/suitesparse.html .

[12] C. Dogbé, Modeling crowd dynamics by the mean-field limit approach. Math. Comput. Modelling 52 (2010), no. 9-10, 1506-1520.

[13] D. Gomes and J. Saude, Mean field games - A brief survey. Dyn. Games Appl. 4 (2014), no. 2, 110-154.

[14] O. Guéant, Mean field games equations with quadratic Hamiltonian: a specific approach, M3AS Math. Models Methods Appl. Sci. 22 (2012), no.9, 1250022, 37 pp.

[15] O. Guéant, J-M. Lasry and P-L. Lions. Mean field games and applications. In ParisPrinceton Lectures on Mathematical Finance 2010, volume 2003, Lecture Notes in Math., Springer, Berlin, 2011.

[16] M. Huang, R.P. Malhamé, and P.E. Caines, Large population stochastic dynamic games: closed-loop McKean-Vlasov systems and the Nash certainty equivalence principle. Commun. Inf. Syst. 6 (2006), no. 3, 221-251.

[17] A. Lachapelle, J. Salomon, and G. Turinici, Computation of mean field equilibria in economics, Math. Models Methods Appl. Sci. 20 (2010), 567-588.

[18] J-M. Lasry and P-L. Lions, Mean field games, Jpn. J. Math., 2 (2007), no. 1, 229-260.

[19] V. Lescarret and E. Zuazua, Numerical approximation schemes for multi-dimensional wave equations in asymmetric spaces. Math. Comp. 84 (2015), no. 291, 119-152.

[20] D. Mercier and S. Nicaise, Existence results for general systems of differential equations on one-dimensional networks and prewavelets approximation, Discrete Contin. Dyn. Syst. 4 (1998), no. 2, 273-300.

[21] S. Nicaise, Elliptic operators on elementary ramified spaces. Integral Equations Operator Theory 11 (1988), no. 2, 230-257.

[22] J. A. Sethian, Level Set Methods and Fast Marching Methods. Evolving Interfaces in Computational Geometry, Fluid Mechanics, Computer Vision, and Materials Science. Cambridge Univ. Press, Cambridge 1999. 